\title{
Assessing reproducibility of inherited variants detected with short-read whole genome sequencing
}

Bohu Pan ${ }^{1 \dagger}$, Luyao Ren ${ }^{2,3+}$, Vitor Onuchic ${ }^{4 \dagger}$, Meijian Guan ${ }^{5 \dagger}$, Rebecca Kusko ${ }^{6 \dagger}$, Steve Bruinsma ${ }^{4}$, Len Trigg ${ }^{7}$, Andreas Scherer ${ }^{8,9}$, Baitang Ning ${ }^{1}$, Chaoyang Zhang ${ }^{10}$, Christine Glidewell-Kenney ${ }^{4}$, Chunlin Xiao ${ }^{11}$, Eric Donaldson ${ }^{12}$, Fritz J. Sedlazeck ${ }^{13}$, Gary Schroth ${ }^{4}$, Gokhan Yavas ${ }^{1}$, Haiying Grunenwald ${ }^{4}$, Haodong Chen ${ }^{14}$, Heather Meinholz ${ }^{4}$, Joe Meehan ${ }^{1}$, Jing Wang ${ }^{15}$, Jingcheng Yang ${ }^{2,3}$, Jonathan Foox ${ }^{16}$, Jun Shang ${ }^{2,3}$, Kelci Miclaus ${ }^{5}$, Lianhua Dong $^{15}$, Leming Shi ${ }^{2,3}$, Marghoob Mohiyuddin ${ }^{17}$, Mehdi Pirooznia ${ }^{18}$, Ping Gong ${ }^{19}$, Rooz Golshani ${ }^{4}$, Russ Wolfinger ${ }^{5}$, Samir Lababidi ${ }^{20}$, Sayed Mohammad Ebrahim Sahraeian ${ }^{17}$, Steve Sherry ${ }^{11}$, Tao Han ${ }^{1}$, Tao Chen ${ }^{1}$, Tieliu Shi ${ }^{21}$, Wanwan Hou ${ }^{2,3}$, Weigong Ge ${ }^{1}$, Wen Zou', Wenjing Guo ${ }^{1}$, Wenjun Bao ${ }^{5}$, Wenzhong Xiao ${ }^{22}$, Xiaohui Fan ${ }^{23}$, Yoichi Gondo ${ }^{24}$, Ying Yu ${ }^{2,3}$, Yongmei Zhao ${ }^{25}$, Zhenqiang Su ${ }^{26}$, Zhichao Liu', Weida Tong ${ }^{1}$, Wenming Xiao ${ }^{27}$, Justin M. Zook ${ }^{28^{*}}$, Yuanting Zheng ${ }^{2,3^{*}}$ and Huixiao Hong ${ }^{1 *}$ (D)

* Correspondence: justin.zook@nist. gov; zhengyuanting@fudan.edu.cn; huixiao.hong@fda.hhs.gov ${ }^{\dagger}$ Bohu Pan, Luyao Ren, Vitor Onuchic, Meijian Guan and Rebecca Kusko contributed equally to this work.

${ }^{28}$ Material Measurement Laboratory, National Institute of Standards and Technology, Gaithersburg, MD 20899, USA

${ }^{2}$ State Key Laboratory of Genetic Engineering, School of Life Sciences and Shanghai Cancer Center, Fudan University, Shanghai 200438, China ${ }^{1}$ Division of Bioinformatics and Biostatistics, National Center for Toxicological Research, US Food and Drug Administration, Jefferson, AR 72079, USA

Full list of author information is available at the end of the article

\begin{abstract}
Background: Reproducible detection of inherited variants with whole genome sequencing (WGS) is vital for the implementation of precision medicine and is a complicated process in which each step affects variant call quality. Systematically assessing reproducibility of inherited variants with WGS and impact of each step in the process is needed for understanding and improving quality of inherited variants from WGS.

Results: To dissect the impact of factors involved in detection of inherited variants with WGS, we sequence triplicates of eight DNA samples representing two populations on three short-read sequencing platforms using three library kits in six labs and call variants with 56 combinations of aligners and callers. We find that bioinformatics pipelines (callers and aligners) have a larger impact on variant reproducibility than WGS platform or library preparation. Single-nucleotide variants (SNVs), particularly outside difficult-to-map regions, are more reproducible than small insertions and deletions (indels), which are least reproducible when $>5 \mathrm{bp}$. Increasing sequencing coverage improves indel reproducibility but has limited impact on SNVs above 30x.
\end{abstract}

Conclusions: Our findings highlight sources of variability in variant detection and the need for improvement of bioinformatics pipelines in the era of precision medicine with WGS. 


\section{Background}

Inherited variants drive susceptibility to diseases spanning oncology [1], central nervous system [2], inflammatory [3], autoimmune [4], and rare diseases [5] plus many more. Reproducible detection of inherited variants enables a better translation of findings from genetic studies into clinical practice via disease diagnosis [1], disease risk assessment [6], and drug development [7]. Whole genome sequencing (WGS) is increasingly used for inherited variant detection due to decreasing cost, single-nucleotide level resolution of nearly the entire human genome, and decreased error rates [8]. However, accurate WGS inherited variant calling is confronted by many challenges. The human genome contains regions of varying complexity, meaning that robust calling in some regions is more difficult than others [9]. Adding to this challenge, sequencing coverage is often uneven across the genome, particularly for targeted sequencing [10, 11]. Regions with more coverage by correctly mapped reads result in more confident calling [10, 12]. Library preparation and sequencing chemistry itself can produce errors, and if these errors accumulate, they lead to false variant calls [13]. Although aligners and variant callers have undergone great improvements in recent years, this process remains error prone, especially in highly repetitive genome regions. Understanding inherited variant reproducibility issues will lead to improved quality for future WGS studies.

To date, efforts such as the Genome in a Bottle Consortium (GIAB) [14], Platinum Genomes Project (PG) [15], and Syndip [16] have produced benchmark or "truth" variant calls and regions against which bioinformatics pipelines can be compared and tested, using publicly available cell lines for GIAB and PG. The Global Alliance for Genomics and Health (GA4GH) recently published a framework for benchmarking variant calling, including standardization of performance metrics [17]. The precisionFDA held two public challenges in 2016 (https://precision.fda.gov/challenges/) for comparing performance of various inherited variant calling pipelines ("consistency" challenge and "truth" challenge). Moreover, several previous studies focused on investigating the impact of potential factors including platform [18-20] or pipeline [21, 22] on genomic variants calling. However, a systematic examination of these factors, together with sequencing platforms, labs, replicates, and DNA samples of different populations is lacking. Here, we seek to further characterize the role of bioinformatics pipelines and interaction with upstream wet lab performance on inherited variant calling. We sequenced triplicates of genomic DNA samples from a Caucasian HapMap trio [23], a well-characterized Chinese quartet from The Quartet Project for Quality Control and Data Integration of Multi-omics Profiling (http://chinese-quartet.org/), and NA12878 used in GIAB [24] using various library preparation kits and sequencing instruments in multiple labs. Inherited variants were called with combinations of multiple aligners and callers.

Via combinations of wet lab experimental and bioinformatics approaches, we assessed the impact of factors involved in variant detection with WGS and their interactions on variant reproducibility for both small variant and structural variant [25]. We found that current sequencing wet lab components including sample preparation, library generation, and sequencing (platform and labs) are much more reproducible than bioinformatics components such as alignment and variant calling, demonstrating the key need of improving bioinformatics analysis in WGS for precision medicine. Our findings highlight the importance of harmonization and could enhance inherited variant calling research across diseases, therapeutic areas, and institutions. 


\section{Results}

\section{Study design and data generation}

We designed a study (Fig. 1 a) to systematically evaluate the reproducibility of inherited variants detected with short-read WGS. More than 109 billion short reads at various coverages were generated from eight DNA samples including a Chinese quartet (CQ-5, CQ-6, CQ-7, and CQ-8), a HapMap trio (NA10385, NA12248, and NA12249), and NA12878 used in GIAB [24] using multiple sequencing platforms and library preparations at different sequencing labs (Fig. 1 c, Additional file 1, Table S1). Fifty-six combinations of different aligners and callers (Additional file 2, Table S2) were used to call SNVs and small indels (Additional files 3-5, Tables S3-S5). The variants were used to assess reproducibility, spanning factors such as sequencing platform/lab/library preparations, alignment, and calling. The variants without filtering (all called variants in Fig. 1 a) were used for evaluation of the reproducibility of variants in all genomic regions without any filtering, hereafter termed as the lower bound of reproducibility.

a

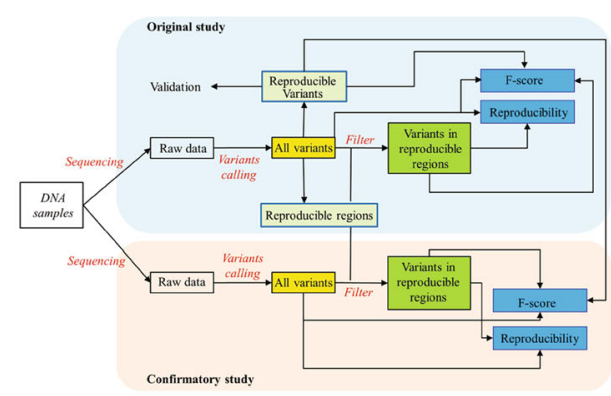

C

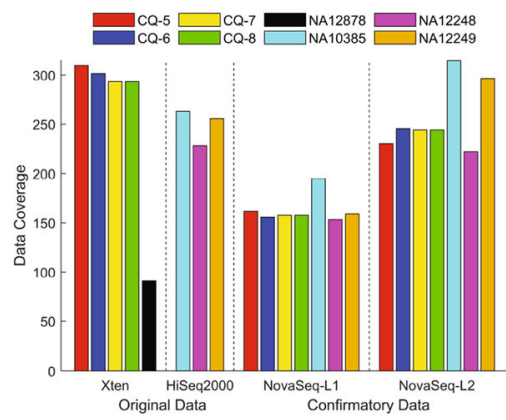

e

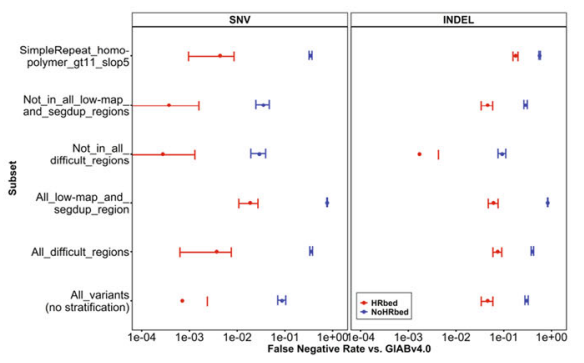

b

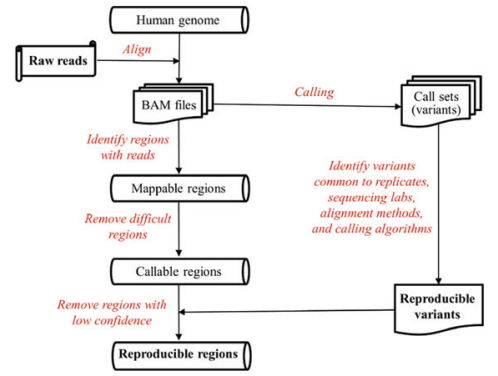

d

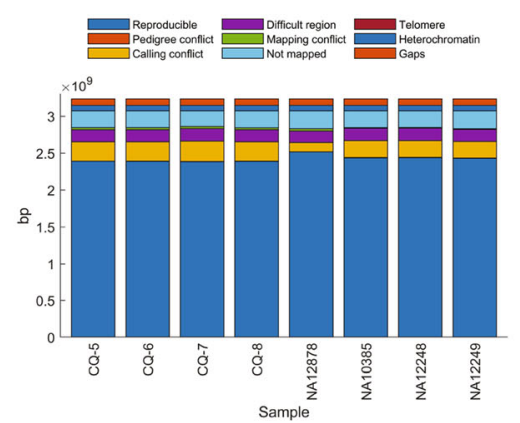

f

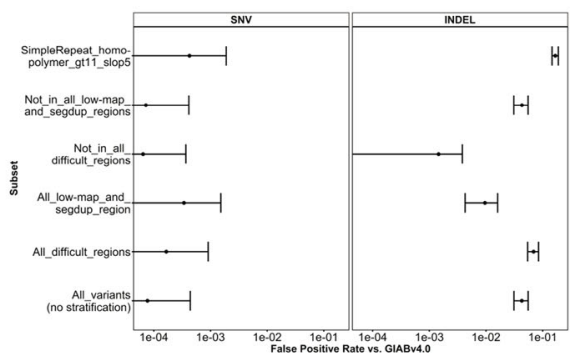

Fig. 1 (See legend on next page.) 
(See figure on previous page.)

Fig. 1 Study design and highly reproducible regions (HRR). a Study design. The DNA samples are from the Chinese quartet, the HapMap trio, and NA12878. WGS was conducted on the samples using different platforms and library preparation kits in multiple labs in the original study (light blue background) and confirmatory study (light brown background). Various variant calling pipelines were employed to generate variants (yellow boxes) from the raw sequence data. The variants were leveraged to define the HRR and pinpoint HRVs (light green boxes). Reproducibility (blue boxes) was analyzed for both all variants and the variants only in HRR (green boxes) in both original and confirmatory studies. The variants with and without HRR-filtering were compared with the HRVs to calculate F-scores (blue boxes), which were used to evaluate reproducibility from a different angle. b Process for defining HRR. All alignment results for the same sample were first examined to find the genomic regions that have sequence reads mapped. Difficult regions such as repeats were then removed to form the callable regions. At last, the HRVs obtained from comparative analysis on all call sets were used to remove the low confidence calling regions from the callable regions, resulting the HRR. c Data generated. Sequencing data coverage is on the $y$-axis for DNA samples. Original and confirmatory data sets are separated with the vertical solid line and depicted with the $x$-axis label. The four Illumina sequencing platforms are separated with the vertical dashed lines and marked on the $x$-axis ticks where L1 indicates the Nextera DNA Flex library preparation kit and L2 is the TruSeq DNA PCR-Free Library Prep Kit. The color legend indicates samples. $\mathbf{d}$ Sizes ( $y$-axis) of HRR (dark blue bars) for the 8 samples (x-axis). The color legend shows the excluded genomic regions, including gap region (dark brown) not in GRCh38, heterochromatin (blue) for condensed DNA labeled as $N$ in the reference, telomere (dark purple) for repeat sequence at the end of the chromosome, not mapped region (light blue), mapping conflict region (green), difficult region (purple) for repeat regions

("SimpleRepeat_imperfecthomopolgt10_slop5.BED" and "remapped_superdupsmerged_all_sort.BED") defined by GA4GH and GIAB, calling conflict region (yellow) for the flanking region of discordant variants, and pedigree conflict region (brown). e False negative rates (FN/(TP + FN)) of HRVs for NA12878 against the GIAB v4.0 benchmark set and stratified by genome context for SNVs (the left panel) and indels (the right panel) in the entire v4.0 benchmark regions (blue) and confined to the HRR (red). Error bars indicate $95 \%$ confidence intervals. $\mathbf{f}$ False positive rates (FP/(TP + FP)) of HRVs stratified by genome context in the entire v4.0 benchmark regions. Error bars indicate 95\% confidence intervals

To estimate the upper bound, highly reproducible variants (HRVs) (Additional file 6, Table S6) and corresponding highly reproducible regions (HRR) (Additional file 7, Table S7) were defined for the eight samples (Fig. $1 \mathrm{~d}$ ) using our workflow (Fig. 1 b, Additional file 8: Fig. S1). Of the highly reproducible SNVs, 1.52 to $1.57 \%$ were in coding regions (Additional file 8: Fig. S2), indicating SNVs from the coding and noncoding regions do not have substantial differences as the size of coding regions accounts for a similar fraction (approximately 1.5\%) of whole human genome [26]. The $\mathrm{G} / \mathrm{C}$ frequencies of the highly reproducible SNVs are markedly higher than those of human genome, consistent with the findings from the international HapMap Consortium and the 1000 Genomes Project [27]. Moreover, the G/C content of the highly reproducible SNVs in coding regions is higher than non-coding regions, which is supported by the fact that coding regions contain a higher $\mathrm{G} / \mathrm{C}$ content than non-coding regions [28]. In contrast, a higher fraction of insertions (Additional file 8: Fig. S3) and deletions (Additional file 8: Fig. S4) are in non-coding regions compared with SNVs, perhaps due to fewer homopolymer and tandem repeats as well as selection against truncating indels in coding regions [29]. Intriguingly, insertions and deletions are comparatively G/Cpoor, especially for non-coding regions. This may reflect the increased indel rate in homopolymers, since A/T homopolymers are more common in the human genome than G/C homopolymers.

All called variants were filtered using the HRR and the resulting variants in the reproducible regions were used to assess the upper bound of reproducibility (Fig. $1 \mathrm{a}$ ). In addition, all called variants and the variants in reproducible regions were compared 
with the HRVs to calculate F-scores. These F-scores then were used to evaluate reproducibility.

To confirm the observed trends in reproducibility from our original study, the same DNA samples were whole genome sequenced using a different Illumina sequencing platform (Illumina NovaSeq) and different library preparations (Illumina Nextera DNA Flex, "Nextera" hereafter, and Illumina TruSeq, "TruSeq" hereafter). Inherited variants were called from the confirmatory sequencing data using the same bioinformatics pipelines. Both lower and upper bounds of reproducibility from the confirmatory study were evaluated in the same way (Fig. 1 a).

\section{Impact of variant class and genome context on reproducibility}

To understand the characteristics of the HRVs, we used GA4GH Benchmarking tools [17] to compare the HRVs to a new v4.0 draft GIAB benchmark for NA12878 [30], which uses long reads and linked reads to make calls in more difficult regions. The recall (or sensitivity) of the HRVs clearly varies by variant size and genome context (Fig. 1 e). The HRVs matched 97.3\% of benchmark SNVs after (vs. 91\% before) excluding all GA4GH-defined difficult regions and complex variants [31], and the false positive (FP) rate is very low at $0.007 \%$ (Fig. $1 \mathrm{f}$ ). Most of the SNVs that were not highly reproducible were in regions difficult to map with short reads and in segmental duplications, since 174,721 of the 281,232 false negative (FN) SNVs fall in these regions. FNs also were highly enriched in $\mathrm{L} 1 \mathrm{H}$ regions $>500 \mathrm{bp}$ and $>75 \% \mathrm{G} / \mathrm{C}$ content. For indels, the FN rate was higher $(\sim 30 \%)$, because, in addition to difficult to map regions, there are several categories of variants that were not highly reproducible, including homopolymers, tandem repeats, indels $>6 \mathrm{bp}$ in size, and complex variants. In total, 119,882 of $144,151 \mathrm{FN}$ indels were in homopolymers or tandem repeats, including 77,083 in homopolymers longer than $6 \mathrm{bp}$ or imperfect homopolymer longer than $10 \mathrm{bp}, 35,266$ in tandem repeats shorter than $51 \mathrm{bp}, 15,992$ in tandem repeats $51 \mathrm{bp}$ to $200 \mathrm{bp}$ long, and 4014 in tandem repeats longer than 200 bp (Additional file 9, Table S8). More FNs likely exist outside the $\mathrm{v} 4.0$ benchmark regions, since it still excludes many long homopolymers, short tandem repeats, and variable number tandem repeats. For indels, the FP rate was higher $(\sim 4.3 \%)$, with most FPs occurring in complex variants, particularly in homopolymers and tandem repeats. The indel FP rate was $<0.2 \%$ after excluding difficult regions and complex variants. Within the HRR for NA12878, the SNV FN rate was $0.07 \%$, SNV FP rate was $0.008 \%$, indel FN rate was $4.6 \%$, and indel FP rate was $4.3 \%$, though if genotype errors were excluded then the indel FN was $0.3 \%$ and the indel FP rate was $0.04 \%$.

\section{Factors impacting reproducibility}

Multiple factors including caller [21, 22], aligner [32, 33], sequencing platform/lab/library $[18,20,34]$ preparation (combined in analysis and simply termed as "platform" hereafter), and sample could affect reproducibility of inherited variants. To assess the impact of these factors, average Jaccard index values among the inherited variants from triplicate DNA samples were first calculated and then analyzed using gradient boosted classification tree to evaluate the contributions of these factors to the trees (Additional file 10, Table S9). For variants with and without HRR filtering, more than $60 \%$ of 
contributions came from callers. Aligners were the second largest contributor (Fig. 2 a). Sequencing platform was the third largest contributor, contributing to more variability for insertions and deletions than SNVs (Additional file 10, Table S9). DNA samples had limited impact on reproducibility, suggesting that any of these eight DNA samples could be used for assessing reproducibility. The observed impacts from the original study were replicated in the confirmatory study (Fig. 2 a). Interestingly, the contributions of caller, platform, and caller $\times$ platform in the confirmatory study were much larger than in the original study. This might be caused by the difference in library preparation kits included in the combined factor platform. All original data were generated using the same library kit TruSeq, while the confirmatory data were generated using two library kits TruSeq and Nextera. Thus, the impacts of platform and caller $\times$ platform on the confirmatory data are larger than on the original data. As all variances
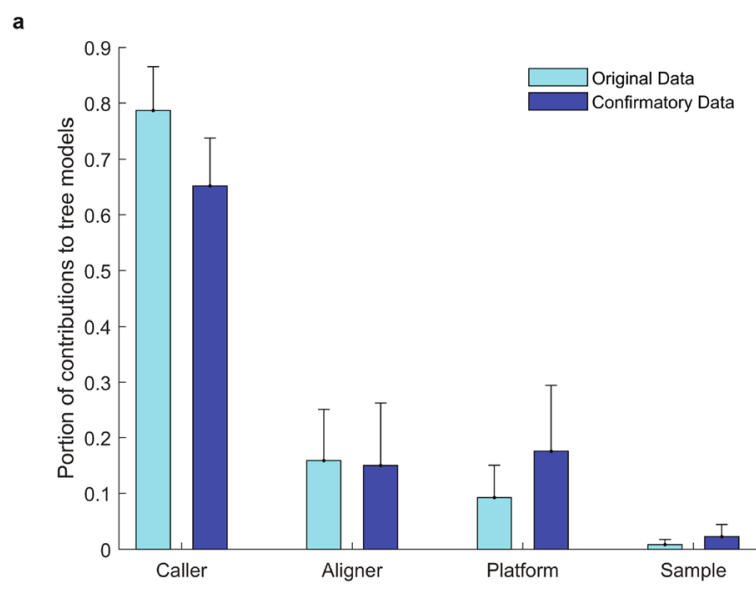

b

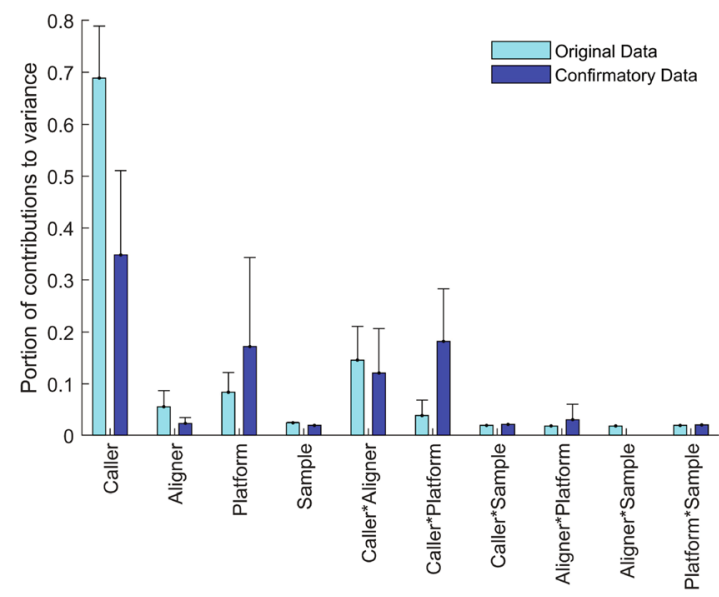

Fig. 2 Impacts of factors on variant reproducibility. a Contributions to gradient boosted trees. The four factors are depicted at the $x$-axis and portions of their contributions to the non-linear gradient boosted tree models are the light blue bars for the original study and are the dark blue bars for the confirmatory study. The error bars are the standard deviations of the portions from different data sets (Additional file 14, Table S13). $\mathbf{b}$ Contributions to reproducibility in variance. The contributions of the four factors as well as their 2way interactions (depicted at $x$-axis) from variance components analysis are plotted as light blue bars for the original study and dark blue bars for the confirmatory study. The error bars are standard deviations of the portions from different data sets (Additional file 15, Table S14) 
for original data or confirmatory data were summed up to $100 \%$, the relative caller impact on the confirmatory data was decreased due to the increase in impacts of platform and caller $\times$ platform. This observation indicates that the contribution of platform to variability may depend on the spectrum of sequencing instruments and library preparations tested.

The gradient boosted classification tree analysis did not separate the impact of interactions between these factors. To further ascertain sources of variance in reproducibility, joint effects of these factors were examined using variance component analysis (Additional file 11, Table S10). The impacts of these factors were in the same order as obtained from the gradient boosted tree analysis: caller $>$ aligner $>$ platform $>$ DNA sample (Fig. 2 b). Furthermore, caller had a large joint effect with aligner. Intriguingly, library preparation (for the confirmatory study) and caller had a considerable joint effect, especially for indels (Additional file 11, Table S10). Again, DNA samples not only had limited contributions to the variance, but also had small joint effects with other factors.

\section{Technical reproducibility}

Analysis of the impact of individual factors on reproducibility was performed to identify potential areas to establish good practices for WGS inherited variant detection. We first assessed technical reproducibility by measuring the concordance of inherited variants between triplicates (Additional file 12, Table S11). The technical reproducibility distributions of SNVs, insertions, and deletions (Additional file 8: Fig. S5-S7) revealed that SNVs were more reproducible than indels. The distributions also indicated a large variation in the technical reproducibility of different calling pipelines. We found that sequencing coverage had limited impact on technical reproducibility of SNVs detected with WGS at $>30 \times$ coverage, while increasing sequencing coverage improved technical reproducibility of indels (Fig. 3 a), especially when sequencing coverage was increased from $30 \times$ to $70 \times$. Both lower and upper bounds of reproducibility of replicate pairs were consistent and not dependent on samples, sequencing platforms, and labs (Additional file 8: Fig. S8-S15).

Variance analysis showed callers and aligners were the two largest components (Fig. 2) but could not reveal performance of individual callers and aligners. Therefore, we examined technical reproducibility values for individual callers and aligners. The aligners performed similarly (Additional file 8: Fig. S16), with Stampy having notably lower technical reproducibility for indels than other aligners, especially with Samtools (Fig. 3 c, d). The technical reproducibility differences between the original and confirmatory studies for SNVs, insertions, and deletions were $0.4 \%$ (95\% confidence: 0.3 to $0.5 \%$ ), $4.4 \%$ (3.8 to $4.9 \%$ ), and $3.7 \%$ (3.2 to $4.2 \%$ ), respectively, providing confidence in the reliability of the technical reproducibility evaluation.

Technical reproducibility comparisons found that the callers had much more variable performance (Additional file 8: Fig. S17), consistent with the variance analysis. More specifically, SNVs from VarScan were less reproducible than SNVs from other callers (Fig. 3 b). Interestingly, VarScan yielded similarly reproducible indels as other callers, while Samtools generated less reproducible indels (Fig. 3 c, d). 
a

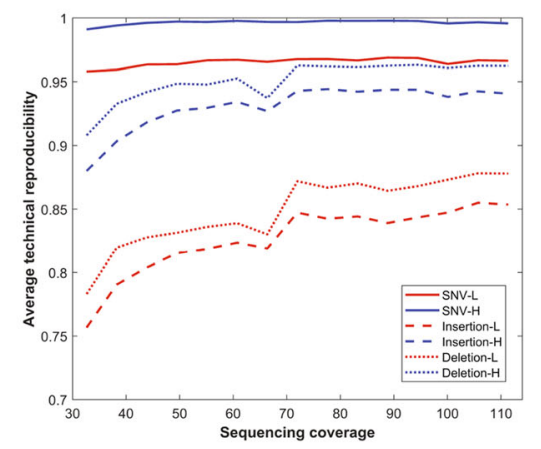

C

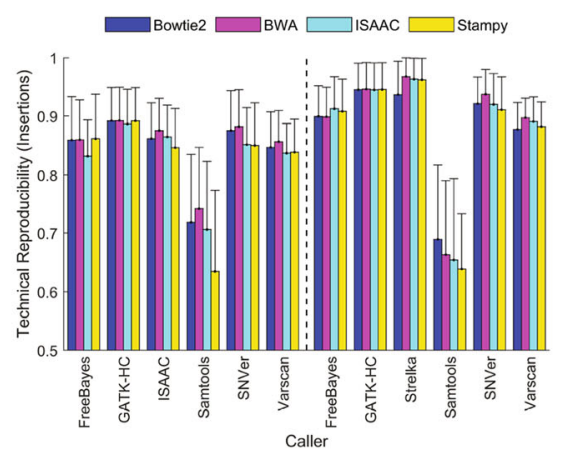

b

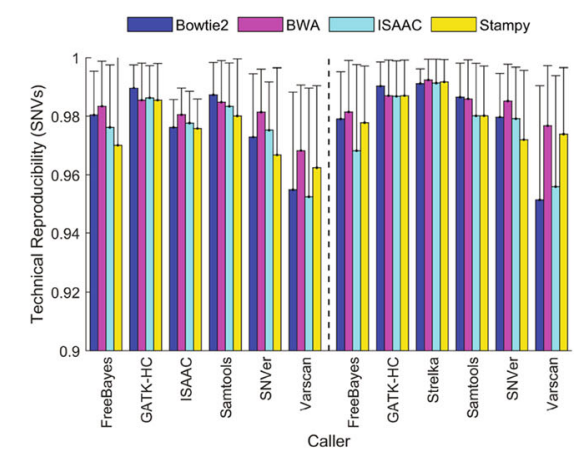

d

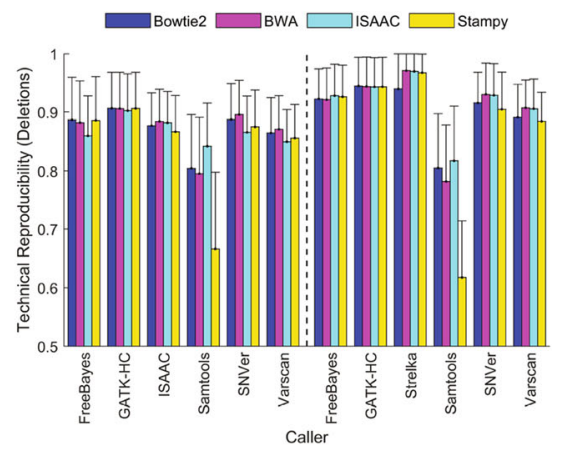

e

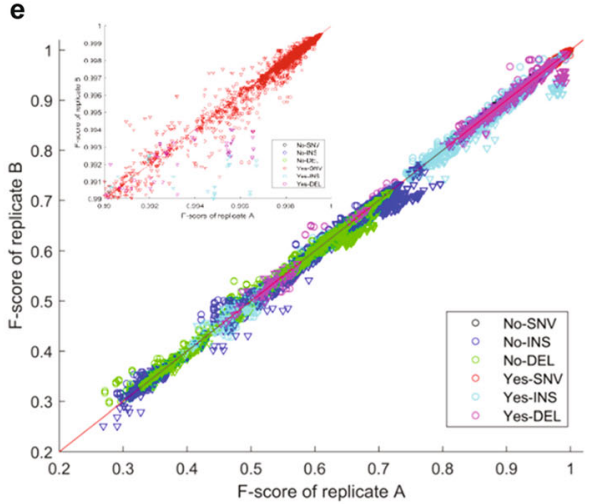

Fig. 3 Technical reproducibility. a Impact of sequencing coverage on technical reproducibility. Average technical reproducibility ( $y$-axis) of detected variants is plotted against the sequencing coverage ( $x$-axis). Line types indicate variant types (SNVs: solid lines, insertion: dash lines, deletion: dot line). Red lines represent upper bounds of technical reproducibility and blue lines are lower bounds of technical reproducibility. b,c,d Technical reproducibility across aligners and callers for SNVs (b), insertions (c), and deletions (d). The average technical reproducibility of variants for pairs of callers ( $x$-axis) and aligners (color legend) are plotted as bars with their standard deviation as sticks. The left panels give the results from the original data and the right panels show the results from the confirmatory data. e F-scores of technical replicates. The F-scores from one technical replicate ( $x$-axis) are plotted against the F-scores from another technical replicate (y-axis). The marker colors represent types of variants indicated at the right bottom corner with two-word text. The first indicates HRR filtering (Yes and No) and the second for variant type (SNV: SNVs, INS: insertions, DEL: deletions). The downward triangles represent the F-scores from the original study, while the circles mark the F-scores from the confirmatory study. The inserted figure at top left is a zoom-in of the F-score $>0.99$ region 
Comparison of the F-scores between the triplicates for SNVs and indels (Fig. 3 e) resulted in similar observations. F-scores of technical replicates were generally reproducible with a correlation coefficient $r=0.993$ and not dependent on samples, variant types, sequencing platforms, and labs as well as calling pipelines.

\section{Lab reproducibility}

To assess lab reproducibility, the Chinese quartet DNA samples were sequenced in three labs in our original study. We calculated reproducibility of the inherited variants detected across the three labs (Additional file 13, Table S12). The lower and upper bounds of lab reproducibility for SNVs were $\sim 0.95$ and $>0.99$ (Fig. 4 a), demonstrating that current WGS methods vary by reproducibility across labs for SNVs. Lab reproducibility for indels was much lower with the lower bound of 0.75 to 0.78 and the upper bound of 0.89 to 0.91 (Fig. 4 a), indicating relatively large room for improvement for

a

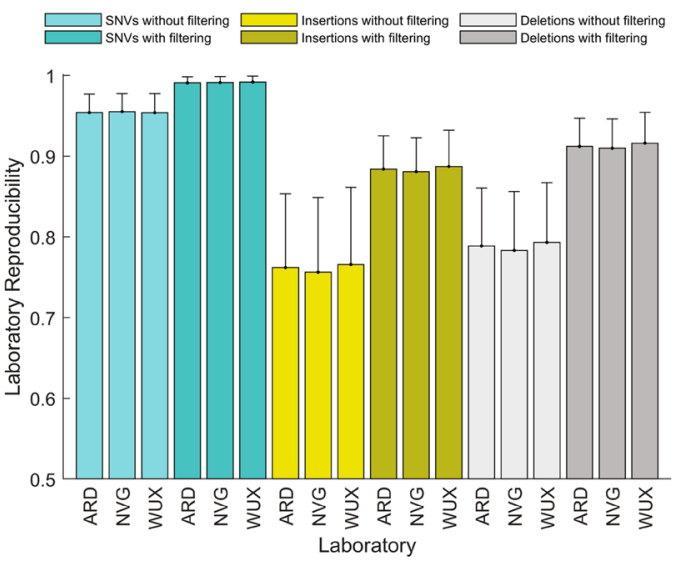

b

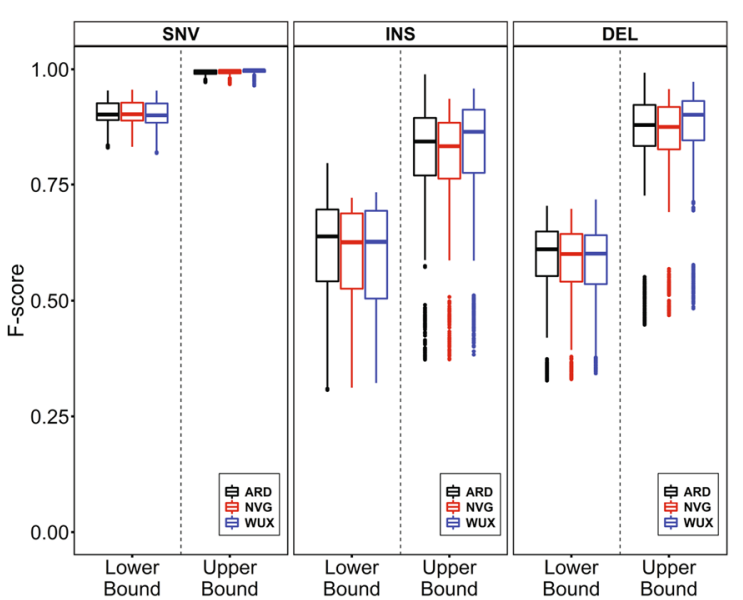

Fig. 4 Lab reproducibility. a Lab reproducibility of the Chinese quartet samples in the original study. The bars represent average values of lab reproducibility and the error sticks indicate standard deviations. The $x$-axis ticks depict sequencing labs. The color legend represents variant types and HRR filtering status. b Boxplots of Fscores for SNVs (left panel), insertions (middle panel), and deletions (right panel). Results from the three labs are plotted in different colors: black for ARD (Annoroad), red for WUX (WuXi NextCODE), and blue for NVG

(NovoGene). F-scores from the lower bound and upper bound of variants are separated and marked at $x$-axis 
indel detection with current WGS. Reproducibility of technical replicates within a lab was consistent among different labs for all three variant types (Additional file 8: Fig. S18 and S19), demonstrating that reproducibility did not vary by lab. To pinpoint causes for the non-reproducible portion, we compared the lab reproducibility for DNA samples, aligners, and callers (Additional file 8: Fig. S20). Consistent with previous work $^{21,22}$, callers were the major cause of lab reproducibility variance, followed by aligners. Reproducibility did not vary much by sample. SNVs from VarScan and indels from Samtools were the least reproducible across the sequencing labs. The reproducibility of variants from different aligners within each lab was similar between labs (Additional file 8: Fig. S21 and S22), further demonstrating that reproducibility did not vary substantially by lab.

Lab reproducibility was also evaluated using F-scores which showed small variations between labs (Fig. 4 b), indicating the dependence of lab reproducibility on other factors (callers, aligners, and platforms). Observations in analysis of the F-scores between labs were consistent with the findings from analysis of variants between labs: lab reproducibility is higher for SNVs, especially for the upper bound, while indels are relatively less reproducible across labs.

\section{Aligner reproducibility}

To ascertain causes of the considerable contributions of aligners (Bowtie2, BWA-MEM (shorten as BWA hereafter), ISAAC, and Stampy) to reproducibility, we calculated variant reproducibility between aligners holding other factors (sample, lab, and caller) constant (Additional file 14, Table S13). The lower bound of aligner reproducibility for SNVs from the original study varied among the aligners, but the upper bound increased from (0.936 to 0.952 ) to (0.994 to 0.998) (Fig. 5 a), demonstrating that SNVs in the HRR were reproducible among aligners. We examined the impact of other factors on aligner reproducibility. Aligner reproducibility appeared independent of DNA samples and sequencing labs but varied with the callers, especially for the variants before filtering to the HRR (Additional file 8: Fig. S23), consistent with the overall variance analysis. Careful examination of aligner reproducibility of the SNVs without filtering found that VarScan and FreeBayes were less reproducible between aligners (0.918 and 0.927, respectively), than ISSAC and GATK-HC (Haplotype caller) (0.967 and 0.961, respectively). However, SNVs in the HRR for all callers reached a high aligner reproducibility $>0.99$ for small variations, indicating that the HRR are useful in identification of reproducible SNVs. Moreover, the observations on aligner reproducibility in the original study were replicated in the confirmatory study (Fig. 5 a).

The lower bound of insertion aligner reproducibility from the original study varied among the aligners ( 0.822 to 0.854 ), while the upper bound increased to 0.936 to 0.952 (Fig. 5 b), demonstrating usefulness of the HRR. DNA samples and sequencing labs did not show substantial differences in aligner reproducibility of insertions. However, callers had a large variation in aligner reproducibility, especially for the lower bound. Comparison between the callers found that Samtools had the lowest aligner reproducibility of insertions, while GATK-HC had the highest aligner reproducibility, possibly due to the local reassembly step in GATK-HC (Additional file 8: Fig. S24). The observations in the original study were confirmed in the confirmatory study. 

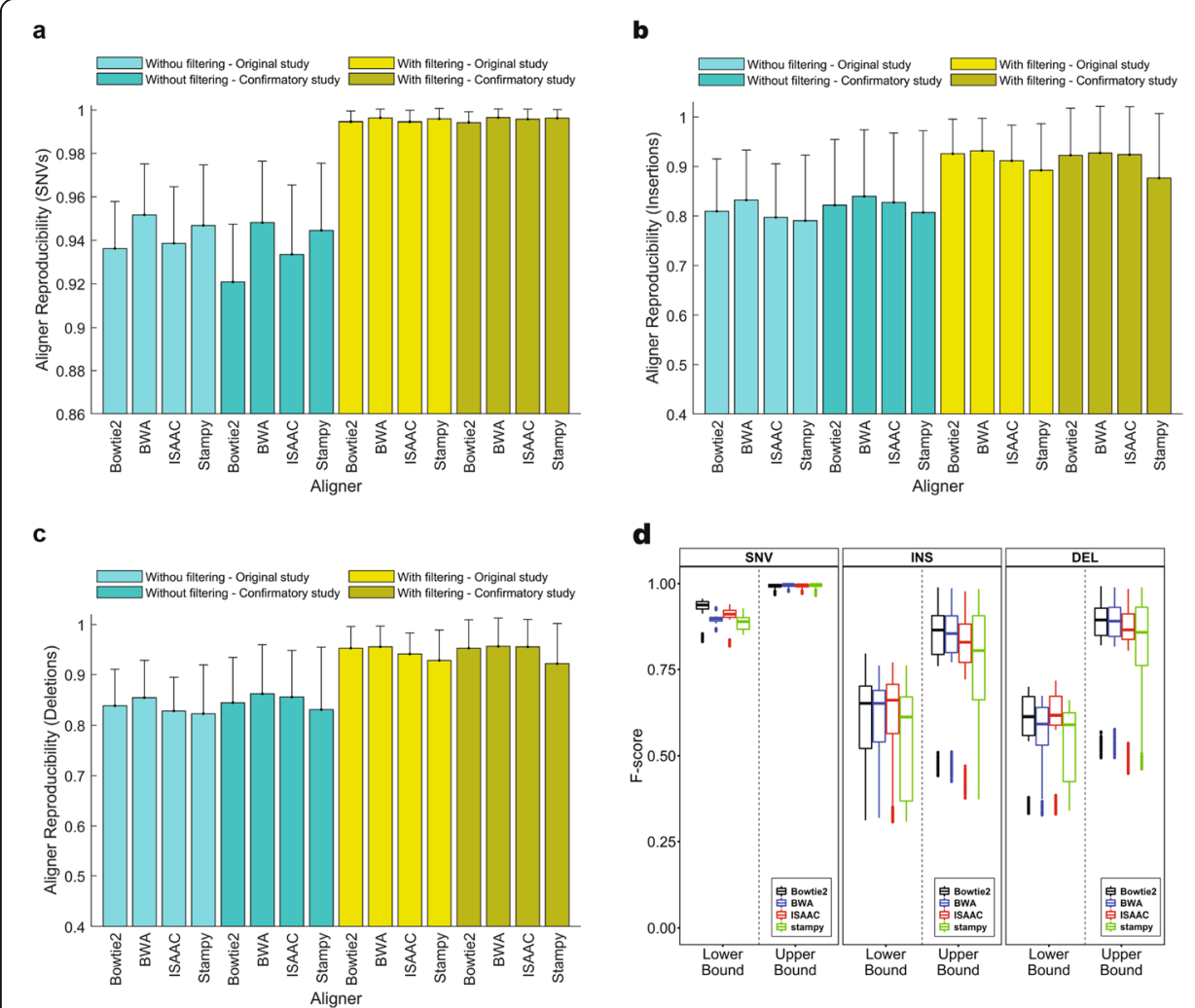

Fig. 5 Aligner reproducibility. a Aligner reproducibility of SNVs. b Aligner reproducibility of insertions. c Aligner reproducibility of deletions. The bars represent average values of aligner reproducibility for the four aligners depicted by the $x$-axis ticks. The error sticks show standard deviation. The color legend specifies if variants were filtered by HRR or not as well as if the data are from original or confirmatory studies. $\mathbf{d}$ Boxplots of F-scores for SNVs (left panel), insertions (middle panel), and deletions (right panel). Results from the four aligners are plotted in different colors: black for Bowtie2, blue for BWA, red for ISAAC, and green for Stampy. F-scores from the lower bound and upper bound of variants are separated and marked at the $x$-axis

The deletions had slightly higher aligner reproducibility than the insertions, but the patterns were similar to insertions (Fig. 5 c). Callers had the largest variation in aligner reproducibility and Samtools had the lowest aligner reproducibility. The patterns of aligner reproducibility for deletions observed in the original study repeated in the confirmatory study (Additional file 8: Fig. S25).

The F-scores between aligners were used to measure aligner reproducibility. The Fscores (Fig. 5 d) indicated that aligner reproducibility was higher for SNVs than for indels. Comparing the lab reproducibility using F-scores (Fig. 4 b) revealed that aligners were less reproducible than labs, indicating aligners caused a relatively larger variation in inherited variants than labs.

\section{Caller reproducibility}

We dissected caller reproducibility in detail to understand the causes of variation. Variants called using different callers were compared to calculate caller reproducibility (Additional file 15, Table S14). The lower bound of caller reproducibility in SNVs from the original study varied from 0.909 to 0.942 , while the upper bound increased to 0.980 to 0.998 (Fig. 6 a). Interestingly, ISAAC in the original study showed a lower 

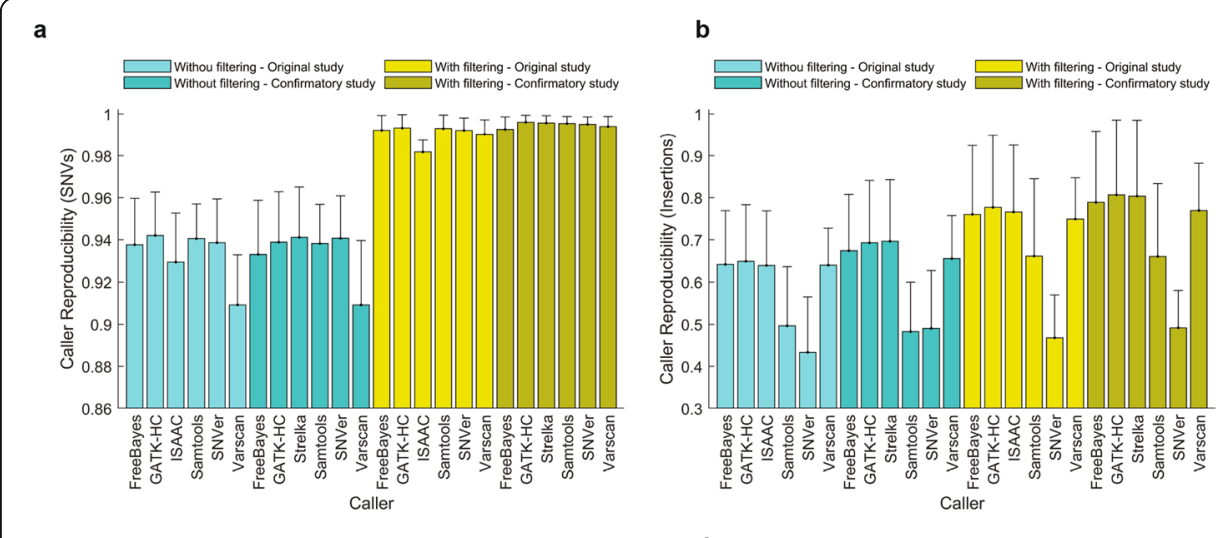

C

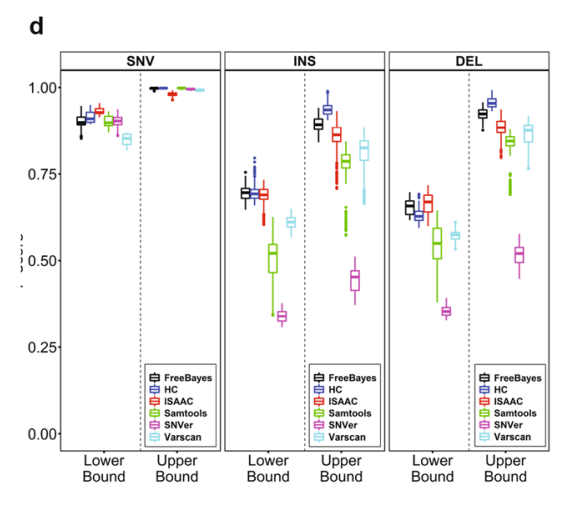

Fig. 6 Caller reproducibility. a Caller reproducibility of SNVs. b Caller reproducibility of insertions. c Caller reproducibility of deletions. The bars represent average values of caller reproducibility for the six callers depicted at the $x$-axis ticks. The error sticks above the bars represent standard deviations. The color legend specifies if variants were filtered by HRR or not as well as data are from original or confirmatory studies. $\mathbf{d}$ Boxplots of F-scores for SNVs (left panel), insertions (middle panel), and deletions (right panel). Results from the six callers are plotted in different colors: black for FreeBayes, blue for HC, red for ISAAC, green for Samtools, magenta for SNVer, and cyan for VarScan. F-scores from the lower bound and upper bound of variants are separated and marked at the $x$-axis

reproducibility than the updated version Strelka2 in the confirmatory study. Examining the impact of other factors found caller reproducibility was affected by aligners, but not by DNA samples and sequencing labs, especially for its lower bound. Compared to aligner reproducibility, caller reproducibility not only was lower but also had a larger variation, consistent with the overall variance analysis (Fig. 2). Bowtie2 and Stampy had a worse lower bound of caller reproducibility in SNVs, while the upper bounds were not substantially different, confirming that the HRR are useful in identification of reproducible SNVs (Additional file 8: Fig. S26). Moreover, the patterns in caller reproducibility in the original study were replicated in the confirmatory study.

Caller reproducibility of insertions (Fig. 6 b) and deletions (Fig. 6 c) not only were lower but also had a larger variation than aligner reproducibility, for both lower and upper bounds. Furthermore, other factors including DNA samples and sequencing labs did not show substantial differences in caller reproducibility for indels (Additional file 8: Fig. S27 and S28). Again, patterns in caller reproducibility for indels in the original study were replicated in the confirmatory study.

The F-scores had a larger variation among the callers (Fig. 6 d) compared with those among aligners, further confirming that callers were the major factor causing variation 
in reproducibility. The F-scores showed that caller reproducibility was higher for SNVs than for indels.

\section{GATK realignment effect}

The old GATK best practices suggested realignment of reads near indels after initial alignment [35]. To evaluate the effect of GATK realignment, we generated variants with and without realignment and then compared their reproducibility. We found GATK realignment did not substantially improve SNV reproducibility across technical replicates (Additional file 8: Fig. S29-S32), labs (Additional file 8: Fig. S33 and S34), aligners (Additional file 8: Fig. S35-S38), and callers (Additional file 8: Fig. S39-S42), indicating benefit of GATK realignment on variant reproducibility is limited. Considering its computational cost, we recommend removal of realignment from variant calling, consistent with the new GATK best practices. Therefore, we did not include it in our confirmatory study.

\section{Discussion}

Inherited variants underpin diseases ranging from rare diseases [36], autoimmune [37, 38], inflammatory conditions [39], developmental disorders [40, 41], and certain familial cancer types [42]. The urgent unmet clinical need in all these disease areas and more precipitates the need for rigorous and robust WGS inherited variant detection. While producing single-nucleotide-level genomic resolution of SNVs and indels, WGS variant calling can be impacted by a multitude of wet lab, sequencing, reference genome [43], and computational factors, as well as interactions of these factors. To fully understand and measure the impact of these factors and to improve the reproducibility of WGS variant calling, it is imperative to comprehensively analyze the relative importance of these sources of variability on inherited variant calling results. In order to understand sources of variation and which variants and regions are most reproducible, we defined the HRVs and HRR for each DNA sample. Furthermore, we performed a confirmatory study with different short-read WGS platforms, library preparations, and bioinformatics methods to replicate the findings in our original study. Consistent results imply that the observed reproducibility could be extendable to other short-read WGS-based methods.

By comparing the HRVs to the GIAB benchmark for NA12878, we found that the HRVs had a low FP rate. However, approximately $91 \%$ of SNVs in the benchmark were reproducible across all the short-read WGS methods we tested, but about 97\% of SNVs were reproducible after excluding all difficult, repetitive regions defined by GA4GH. Most of the non-reproducible SNVs were in difficult to map regions. Approximately $30 \%$ of indels were not reproducible, particularly for indels $>5 \mathrm{bp}$. These results highlight limitations of short-read technology and the importance of optimizing bioinformatics in difficult to map regions and for large indels.

Short-read sequencing is notably limited for tandem repeats longer than the read length and segmental duplications [44, 45]. Thus, we excluded these regions in the HRR. Both sequencing technology and calling algorithms will need to improve to increase the reproducibility of variants in these regions in the future [46]. Development of long-read sequencing technology of $\sim 1000 \mathrm{bp}$ or more with higher base-call 
precision could be ideal for variant detection in such repetitive regions [47, 48]. Moreover, the observation of a large distance between the upper and lower bounds of reproducibility, especially for indels (Fig. 3 A) suggests caution when considering inherited variants detected outside of the HRR with new algorithms or technologies.

We systematically evaluated the impact of several factors in WGS inherited variant detection. Our results revealed that bioinformatics pipelines had the largest impact on variant calling reproducibility. Breaking bioinformatics up into components, the caller contributed more than the aligner to the variance in reproducibility. In contrast to the impact of bioinformatics, short-read sequencing experiment-related factors (labs, platforms, library preparations, DNA samples) had much smaller impacts, though they can still be important. Our findings suggest that selection of aligner and caller for inherited variants calling should be done carefully. The somatic mutations working group of SEQC2 also found that bioinformatics components were the largest source of variability in somatic mutations [49]. The specific ranked performance of the bioinformatics tools established here was solely based on reproducibility and thus may not be extrapolated accurately to other studies with focus on accuracy, sensitivity, or efficiency [46]. It is important to understand that reproducibility is no panacea. Even though our results did not show a specific aligner or caller constantly outperform others in reproducibility, we demonstrated that depending on variant types some aligners and callers performed worse than others. Therefore, when setting up bioinformatics pipeline for inherited variant calling from short-read WGS data, Strelka2 and HaplotypeCaller in GATK are recommended to ensure reproducibility. On the other hand, comprehensive comparisons of extensive WGS analyses for inherited variants are directly applicable to most mammalian species that have a comparable size to the human genome with a similar G/C content. Thus, our study should contribute not only to medical and clinical fields but also to fundamental genomic sciences, e.g., from evolutionary studies to disease model animal developments.

For every study, the question arises of how the tradeoff between sensitivity at the potential expense of reproducibility should be handled, depending on the study goal and the resources available. Furthermore, our study clearly highlights the necessity of standardized pipelines, especially for large projects, and the importance of harmonization approaches and continuous quality assessment over controls (e.g., UK-biobank). Nevertheless, it is also apparent from our study that challenges in SNV calling remain, such as the impact of alignment methods that cannot be resolved over a realignment step, or other factors carefully outlined here that lead to variabilities. Ideally, the SNV callers would in the future incorporate models that may make deduplication of reads and such unnecessary and further increase reproducibility and confidence in variation.

To confirm that our observations from the reproducibility analysis continue to be useful for new short-read sequencing platforms and analysis methods, we applied similar methods to the confirmatory sequencing data from new platform or library preparations (TruSeq without PCR and Nextera with PCR) based on our generated HRVs and HRR. We found most individual callers performed similarly as in the original data set. Only ISAAC and its updated version Strelka2 changed in performance. This observed improvement in the confirmatory data set is likely driven by algorithmic updates. Taken together, our results suggest an advantage of selecting a pipeline with continued support and development at the time of setting up the bioinformatics pipeline. As an 
interesting addendum, we also found the PCR-free TruSeq library had similar reproducibility to the PCR-based Nextera library.

We did not include filtering/variant recalibration for GATK-HC. It is worth to point out that variant recalibration is an important source of variability. However, this is a time-consuming variable to explore and requires well-curated training resources and is also not suitable for small-sample-size experiments such as this study. Though VariantFiltration tool in GATK can be used to hard-filter variants called from GATK when variant recalibration is hard to perform, such variant filtering (variant recalibration tool) is beyond the scope of this paper because we focused on reproducibility of variants without post-calling processing for all calling algorithms.

Despite our experimental design, the current study has several limitations. First, our analysis focused on SNVs and small indels. Other types of variants including structural variants, copy number variations, and tandem repeats were kept for future work. Our results were for short-read data and short-read variant callers; thus, our findings may not be extrapolated to long-read technologies and different calling algorithms. Nevertheless, we expect similar challenges, if not more, with the infant state of methods currently available for long reads and the constant updating of sequencing technologies [50]. In this study, we further followed the default/recommended parameters for shortread aligners and callers and their combinations. We focus on the impact of bioinformatics pipelines with their most commonly used default settings on inherited variants calling rather than to optimize and recommend specific parameters for bioinformatics pipelines or performing a comprehensive analysis of each caller itself, since bioinformatics pipelines evolve rapidly.

\section{Conclusions}

In summary, we queried whether various factors in inherited variant detection with WGS including sequencing platform/lab/library, aligner, and caller contribute to variance in reproducibility. The performance of data sets of technical replicates from different sequencing labs, libraries, and bioinformatics components assessed in our study can be used as a reference supporting regulatory science and precision medicine research for the WGS research community. To enable the research community to leverage our work, we provided our raw data and code for defining the HRVs and HRR to the community for making their own tweaks to improve practices for inherited variant detection and to develop more reproducible bioinformatics pipelines.

\section{Methods}

\section{Sequencing of HapMap trio by Illumina HiSeq2000}

The Hapmap Trio DNA samples (NA10835, NA12248, and NA12249) were purchased from Coriell Cell Repositories (Camden, NJ). The concentration and quality were quantified using a NanoDrop 2000c. The OD260/280 ranged from 1.8 to 1.89. The original DNA samples were diluted to $50 \mathrm{ng} / \mu \mathrm{L}$ and $100 \mu \mathrm{L}$ from each sample and were used for Illumina sequencing.

Genomic DNA was quantified prior to library construction using PicoGreen (Quant-iT ${ }^{\mathrm{Tm}}$ PicoGreen ${ }^{\circ}$ dsDNA Reagent, Invitrogen, Catalog \#: P11496). Quants were read with Spectromax Gemini XPS (Molecular Devices). 
Paired-end libraries were manually generated from $500 \mathrm{ng}$ to $1 \mu \mathrm{g}$ of gDNA using the Illumina TruSeq DNA Sample Preparation Kit (Catalog number FC-121-2001), based on the protocol in the TruSeq DNA PCR-Free Sample Preparation Guide. Prefragmentation gDNA cleanup was performed using paramagnetic sample purification beads (Agencourt ${ }^{\circ}$ AMPure ${ }^{\circ}$ XP reagents, Beckman Coulter). Samples were fragmented and libraries were size selected following fragmentation and end-repair using paramagnetic sample purification beads, targeting 300-bp inserts. Final libraries were quality controlled for size using a gel electrophoretic separation system and were quantified.

Following library quantitation, DNA libraries were denatured, diluted, and clustered onto v3 flow cells using the Illumina $\mathrm{cBot}^{\mathrm{tm}}$ system. cBot runs were performed based on the cBot User Guide, using the reagents provided in Illumina TruSeq Cluster Kit v3.

Clustered v3 flow cells were loaded onto HiSeq 2000 instruments and sequenced with $100 \mathrm{bp}$ paired-end, non-indexed runs. All samples were sequenced on independent lanes. Sequencing runs were performed based on the HiSeq 2000 User Guide, using Illumina TruSeq SBS v3 Reagents. Illumina HiSeq Control Software (HCS) and realtime analysis (RTA) was used on HiSeq 2000 sequencing runs for real-time image analysis and base calling.

\section{Sequencing of Chinese quartet and NA12878 by Illumina XTen}

Chinese Quartet reference materials were from four immortalized lymphoblastoid cell lines (LCLs) of a "Chinese Quartet" family including father, mother, and two monozygotic twin daughters. These family volunteers were from the Fudan Taizhou cohort, representing a typical Chinese ethnicity genetic background. Lymphoblastoid cell lines were immortalized from blood B cells using Epstein-Barr virus (EBV) transformation. This study was approved by the independent ethics committee at the School of Life Sciences of Fudan University. All volunteers provided written informed consent to participate in the study.

The LCLs were cultured in RPMI 1640 (Gibco Catalog No. 31870-082) supplemented with fetal bovine serum (Gibco 10091-148) to a final concentration of $10 \%$ by volume. Cells were maintained at $37{ }^{\circ} \mathrm{C}$ with $5 \% \mathrm{CO}_{2}$ and were sub-cultured every 3 to 4 days. After six passages, a total of about $2 \times 109$ cells were used for DNA extraction. The collected cells were washed with PBS for twice before DNA extraction using Blood \& Cell Culture DNA Maxi Kit (QIAGEN 13362). The extracted DNA samples were stocked in TE buffer (10 mM TRIS, 1 mM EDTA, pH 8.0).

The NA12878 DNA reference material (RM8398) was purchased from the National Institute of Standards and Technology (NIST).

WGS data for Chinese Quartet and RM8398 reference materials were generated from three sequencing labs (ARD: Annoroad, WUX: WuXi NextCODE, and NVG: NovoGene) using the Illumina XTen machine.

Libraries were prepared for whole genome sequencing using TruSeq DNA nano (Illumina catalog number 15041110) according to the manufacturer's instructions. In total, 200 ng DNA was used for the TruSeq library preparations. All labs unified in-house fragmentation conditions using Covaris with a target size of $350 \mathrm{bp}$. All reference materials were prepared with three replicates in a single batch. The library concentrations were measured by the Qubit 3.0 fluorometer with the Quant- 
iT dsDNA HS Assay kit (Thermo Fisher Scientific, catalog number Q32854). The quality of all libraries was assessed using an Agilent 2100 Bioanalyzer or TapeStation instrument (Agilent).

These whole-genome libraries were sequenced on the Hiseq XTen (Illumina) with paired end $150 \mathrm{bp}$ read length leveraging synthesis (SBS) chemistry. Sequencing was performed following the manufacturer's instructions.

\section{Sequencing of HapMap trio and Chinese quartet by Illumina NovoSeq with library preparation kit Nextera}

Samples were quantified for dsDNA content with the Qubit dsDNA HS assay kit. Out of 21 samples, two contained less than 100 ng DNA. For samples with sufficient DNA, $100 \mathrm{ng}$ was used as input for the Illumina Nextera DNA Flex library preparation kit (Illumina, catalog number 20018704). Libraries were prepared according to the manufacturer's instructions (Illumina, Nextera DNA Flex Library Prep Reference Guide), with five PCR cycles used for amplification. For the two lower input samples, one sample had $62 \mathrm{ng}$ DNA input and was prepared the same as the 100-ng samples. The other sample had $17 \mathrm{ng}$ DNA input, so the PCR cycle number was increased to nine cycles, which resulted in shorter insert sizes in the final library for this sample.

Library yield and fragment size were quantified using the Qubit dsDNA HS assay kit and Agilent 2100 Bioanalyzer HS DNA chip, respectively. Libraries were loaded onto two NovaSeq S4 flow cells and clustered according to manufacturer's instructions. Run data sets were uploaded to BaseSpace, and fastq files were generated.

\section{Sequencing of HapMap Trio and Chinese quartet by Illumina NovaSeq with library preparation kit TrueSeq}

Libraries were prepared using the TruSeq DNA PCR-Free Library Prep Kit (Illumina, catalog number 20015962) with a modified protocol to target 450 bp insert using 600 ng input. Shearing was performed with Covaris LE220 (18\% Duty factor, 450 PIP (W), 200 Cycles/Burst, $60 \mathrm{~s}, 4$ to $8.5^{\circ} \mathrm{C}$ ) and SPRI dilution to remove large DNA fragments $(88 \mu \mathrm{L} \mathrm{SPB}+72 \mu \mathrm{L}$ Water), and IDT for Illumina Unique Dual Indexes (Illumina, catalog number 20020178). Sequencing was performed on the Illumina NovaSeq6000 Sequencing System with Xp loading on an S4 flowcell and $151 \times 8 \times 8 \times 151$ cycles. Raw run data were streamed onto the BaseSpace Sequence Hub from the sequencer. Fastq files were generated using bcl2fastq on the BaseSpace Sequence hub with default parameters. Adapters were trimmed during fastq generation using AGATCGGAAGAG CACACGTCTGAACTCCAGTCA as the read 1 adapter and AGATCGGAAGAGCG TCGTGTAGGGAAAGAGTGT as the read 2 adapter. To confirm quality and coverage of samples, fastqs were processed through the Whole Genome Sequencing v8.0.1 BaseSpace app, with alignment against the GRCh38Decoy reference genome with default parameters. Down sampling to $100 \times$ coverage was enabled in the Whole Genome Sequencing app for any sample with coverage beyond 100x. Two samples in the original NovaSeq 6000 S4 run did not reach $60 \times$ average autosomal coverage and were re-sequenced on an S1 flowcell. Re-sequenced samples were analyzed in the same manner, and we confirmed greater than $60 \times$ coverage. 


\section{Quality assessment of sequencing data}

All fastq files were evaluated with FastQC [51] (v0.11.5) with default setting for assessment of base quality, adapter content, and so on. Per base sequence quality was extracted with shell script from the "fastqc_data.txt" file reported by FastQC to check if the data quality passed or not.

\section{Sequence reads alignment}

The short reads were first aligned to the latest human reference genome [43] (GRCh38 with decoy sequences downloaded from Genomic data commons of the National Cancer Institute) using four aligners: Bowtie2 (v2.2.9) [52], BWA [53] (v0.7.15), ISAAC [54] (v1.0.7), and Stampy [55] (v1.0.29). Default settings for Bowtie2 and BWA were applied. BWA was used as a pre-aligner for Stampy, which was suggested by Stampy's developer for efficient alignment. Stampy's default settings were used except for application of the "--bamkeepgoodreads". The setting “--base-calls-format --stop-at Bam --keep-unaligned back --realign-gaps yes" was used in ISAAC alignment to get sorted BAM files. Resulting SAM files from the other three aligners were sorted and converted to BAM files by the SortSam module in Picard [56] (v2.7.1). Duplicates in the sorted BAM files were marked by module MarkDuplicates and read groups were assigned by module AddOrReplaceReadGroups in Picard (v2.7.1).

\section{GATK realignment}

All BAM files obtained from alignment in the original study were processed with GATK [35] realignment following the best practices recommended by the Broad Institute (Notice: the realignment recommendation was removed by the Broad Institute beginning with GATK v4.0). Each BAM file was processed with local-realignment by GATK modules RealignerTargetCreator and IndelRealigner and base-quality recalibration by GATK modules BaseRecalibrator and PrintReads by following the best practices from the Broad Institute. The known SNPs and indels for GRCh38 in DBsnp146 (ftp:// gsapubftp-anonymous@ftp.broadinstitute.org/bundle/hg38/dbsnp_146.hg38.vcf.gz) and two indel files (ftp://ftp.1000genomes.ebi.ac.uk/vol1/ftp/technical/reference/GRCh38_ reference_genome/other_mapping_resources/Mills_and_1000G_gold_standard.indels.b3 8.primary_assembly.vcf.gz) and (ftp://ftp.1000genomes.ebi.ac.uk/vol1/ftp/technical/ reference/GRCh38_reference_genome/other_mapping_resources/ALL.wgs.1000G_ phase3.GRCh38.ncbi_remapper.20150424.shapeit2_indels.vcf.gz) were used as reference in the realignment and recalibration process.

\section{Variant calling}

BAM files with and without GATK realignment were used for variant calling using six different callers: FreeBayes [57] (v1.1.0), GATK-HaplotypeCaller [35] (v3.7), ISAAC [54] (v 1.0.7), Samtools [58] (v1.3.1), SNVer [59] (v0.5.3), and VarScan [60] (version 2.3.9). The running options "-X $-0-\mathrm{u}-\mathrm{v}$ " in FreeBayes, “-rf BadCigar -dbsnp dbsnp_ 146.hg38.VCF --stand_call_conf 30" in GATK-HaplotypeCaller, "minMapq $=20$; $\operatorname{minGQX}=30$ " in ISAAC, "-ugf" and "-vmO" from bcftools (v1.3.1) in Samtools, "-p 0.05 ” in SNVer, and “-p 0.05 --min-coverage 8 --min-reads2 2 --p-value 0.05” in VarScan were used in variant calling. Variant calling results were stored in VCF format. 


\section{Variant calling by Sentieon}

The decoy version of GRCh38 human reference genome (https:/gdc.cancer.gov/aboutdata/data-harmonization-and-generation/gdc-reference -files; GRCh38.d1.dv1.fa) from the Genomic Data Commons (GDC) was used in variant calling by Sentieon (v201711.02). The Sentieon DNAseq pipeline is a tool for variant calling from raw Fastq files, including read mapping by BWA, duplicate removal, indel realignment, base quality score re-calibration, and variant calling by Haplotyper. The Sentieon DNAseq pipeline, Sentieon [61] v201711.03, provides a complete rewrite of the mathematical models of the GATK Best Practices with a focus on computational efficiency, accuracy, and consistency. In variant calling by Sentieon, sequence reads were first aligned to GRCh38.d1.vd1.fa with Sentieon BWA, followed by sorting and indexing with the Sentieon utility. Subsequently, duplicate reads were removed, and base qualities were recalibrated with the Sentieon driver program. Variant calling was then performed with Sentieon Haplotype caller.

\section{Variant calling by Dragen}

Reads were aligned to human genome reference GRCh38 in BaseSpace using DRAGEN Germline Pipeline version 3.2.8 in Whole Genome Sequencing v7.7.0 (WGSv7). Although the DRAGEN aligner was able to use all reads for alignment, WGSv7 requires fewer than 1 billion paired end reads for analysis. Therefore, fastq files were downsampled to 990 million paired-end reads in BaseSpace using FASTQ Toolkit v2.2.0 to enable WGSv7 analysis. Variant calling was performed in BaseSpace using DRAGEN Germline Pipeline version 3.2.8. The DRAGEN pipeline was run with default settings with "Map/Align + Variant Caller" selected, and CNV calling, SV calling, and duplicate marking enabled.

\section{Variant calling by RTG}

The following describes the processing used to align reads and call variants using RTG [62] alignment and variant calling algorithms.

All FASTQs underwent quality-based filtering to trim off poor quality read ends (using "rtg fastqtrim" --end-quality-threshold 15) and formatting to the RTG SDF format (which allows random access to arbitrary chunks of reads during mapping) using "rtg format". FASTQ file pairs for each replicate were merged to a single per-replicate SDF and assigned a unique read group.

Alignment of the reads for each sample to the reference genome GRCh38 was via "rtg map," processing reads from the input SDF file in chunks to permit partitioning of the alignment across multiple nodes. A typical chunk size was 40 million read-pairs. During alignment, an appropriate pedigree file was supplied to the mapping command to allow the aligner to lookup the sex of the sample. After primary alignment, an additional mate-pair rescue tool (currently in development) was executed on any reads which were unmapped but for which the other arm of the pair was uniquely mapped, and any rescued alignments were included in subsequent variant calling.

Across the various samples and families in the SEQC2 project, several variant calling modes were employed. When calling a single sample in isolation, the "rtg snp" command was used, for example: rtg snp -t GRCh38.d1.vd1.sdf $\backslash$-T 8 --pedigree 
pedigree.ped \--enable-allelic-fraction --XXcom.rtg.variant.mask-homopolymer=true $\backslash$ -o snp_HG001-r1-H3WNJDSXX_S8 \ map_HG001-r1-H3WNJDSXX_S8.sdf_"/alignments.bam. The final argument supplies all the alignment BAMs corresponding to the particular sample.

Analysis of Mendelian inheritance errors were computed using "rtg mendelian." Overall variant statistics for each sample were computed using "rtg vcfstats."

\section{Variance analysis}

Variants concordance was calculated using the average Jaccard Index as follows: $((\mathrm{A} \cap \mathrm{B}) /(\mathrm{A} \cup \mathrm{B})+(\mathrm{A} \cap \mathrm{C}) /(\mathrm{A} \cup \mathrm{C})+(\mathrm{B} \cap \mathrm{C}) /(\mathrm{B} \cup \mathrm{C})) / 3$, where $\mathrm{A}, \mathrm{B}$, and $\mathrm{C}$ are variants from the three replicates of each sample. Contribution of four factors (caller, aligner, platform, and sample) to the variation in concordances was estimated by a non-linear Gradient Boosted Tree (JMP Pro v14.3). The importance of each factor was estimated by how often it is used to make key decisions with decision trees. Boosting is the process of building a large, additive decision tree by fitting a sequence of smaller decision trees, called layers. The tree at each layer consists of a small number of splits. The tree is fit based on the residuals of the previous layers, which allows each layer to correct the fit for poorly fitting data from the previous layers. The final prediction for an observation is the sum of the predictions for that observation over all of the layers. The factor contributions were estimated in the model fitting, which is based on the total number of instances over all of the trees when the specific factor is used to split the data. The proportion of the contribution of each factor was calculated as sum of squares attributed to the factor divided by the total sum of squares.

In addition to the non-linear method, we also estimated the contribution of all possible 2-way interactions of the factors in a Variance Components Analysis (JMP Pro v14.3). The variance components were parameterized using an unrestricted method [63] in a mixed model fitted with restricted maximum likelihood (REML). Student's $t$ test was used to assess the contribution difference between factors. Variance components were estimated through fitting a random effect model as follows:

$$
\begin{aligned}
& Y=Z \gamma+\varepsilon, \\
& \gamma \sim N(0, G) \\
& \varepsilon \sim N\left(0, \sigma^{2} I_{n}\right)
\end{aligned}
$$

where $Y$ denotes an $n \times 1$ vector of response (Jaccard index), $Z$ is the design matrix for the random effects, and $\gamma$ is a vector of unknown random effects with design matrix $Z$. Both $\gamma$ and $\varepsilon$ are assumed following normal distribution with means at $0 . G$ and $\sigma^{2}$ are the variance components that need to be estimated. The ratio of the contribution of each factor to the overall variability was calculated by the variance component of each factor divided by the total.

\section{Generation of highly reproducible variants (HRV) and highly reproducible regions (HRR)}

We generated HRVs and defined HRRs to assess the upper bound of reproducibility based on the suggestion from GIAB4. We used the workflow shown in Additional file 8: Fig. S1 to generate the HRRs. Detailed procedures for defining the HRRs are given below. 


\section{Determining mappable regions}

Each of the BAM files from all aligners (with and without GATK realignment), replicates, and labs was used to generate a region file having aligned reads using GATKCallableLoci with cutoffs of minimum depth of 6, maximum depth of 160, minimum mapping quality of 10 , and minimum base mapping quality of $20 \%$. We created 81 and 27 region files for each Chinese Quartet sample and HapMap sample (including NA12878), respectively. The mappable regions for each sample were determined as the genome regions that were covered by any of the region files of the sample. Technically, the mappable regions for a sample are the union of the region files.

\section{Identify consensus mappable regions}

The determined mappable regions for a sample were covered by a different number of region files. To identify the consensus mappable regions of the sample, its determined mappable regions were ranked by the number of region files that cover the regions. The top 99\% ranked determined mappable regions were elected as the consensus mappable regions. The resulting consensus mappable regions are the regions covered by $\geq$ 10 region files for CQ-5, CQ-6, and CQ-7, by $\geq 11$ region files for CQ-8, by $\geq 9$ for NA12878, and $\geq 3$ for all three HapMap samples.

\section{Determine callable regions}

Some genomic regions present variant calling difficulties and were removed from the identified consensus mappable regions. Specifically, simple repeats including homopolymer regions and super duplications defined in "SimpleRepeat_imperfecthomopolgt10_ slop5.bed" and "remapped_superdupsmerged_all_sort.bed" by GIAB and GA4GH were removed using the subtract command from bedtools. The remaining consensus mappable regions were determined to be callable regions.

\section{Define HRVs}

First, the variants called from the same pipelines for three replicates of the same sample were compared and the variants called in only one replicate were filtered out as discordant variants. The remaining variants were used as replicateconcordant variants for the sample. Comparing the replicate-concordant variants from the three labs for the Chinese Quartet samples further filtered discordant variants that were found in the replicate-concordant variants of only one lab. The replicate-concordant variants of the HapMap samples and NA12878 as well as the post-filter replicate-concordant variants of the Chinese Quartet samples were then compared among aligners to determine aligner-concordant variants by filtering discordant variants that were identified in the replicate-concordant variants from only one aligner. The aligner-concordant variants were further compared among callers by filtering discordant variants that were shared by six or less callers. The remaining aligner-variants were determined as caller-concordant variants. The caller-concordant variants for NA12878 were defined as HRVs. The callerconcordant variants for the twins of Chinese Quartet were compared to filter discordant variants between the twins and the remaining caller-concordant variants were used for Mendelian rule compliance checking together with the call- 
concordant variants of the parent samples of Chinese Quartet and the HapMap trio samples. Variants violating the Mendelian rule were filtered out as discordant variants and the remaining Mendelian rule compliant variants were defined as HRVs.

\section{Defining HRRs}

For each sample, its HRVs and all discordant variants were used to filter the callable regions. For each of the discordant variants, the genome region $50 \mathrm{bp}$ to its left and $50 \mathrm{bp}$ to its right was compared with HRVs. If no HRV was located in this region, this region was removed from the callable region. When this region had HRVs, half of the region between the discordant variant and the nearest HRV were removed. After removal of such regions for all discordant variants, the remaining callable regions were defined as the HRR of the sample.

\section{Filtering variants from different pipelines}

Different callers report variants with different minimum read depths. We applied depth filtering prior to lower bound reproducibility calculation so that the variants in reproducibility calculation have the same minimum read depth. We also filtered variants with very high read depth using a cutoff of mean read depth plus three times standard deviation of the read depth of all variants. Specifically, we used a minimum read depth of 8 for all samples and a maximum read depth of 223 for the Chinese quartet samples and NA12878 and a maximum read depth of 350 for HapMap trio samples. To assess the upper bound of reproducibility, we selected variants only in HRR. Specifically, the vcffilter command of RTG tool was used to filter the variants outside HRR.

\section{Reproducibility calculation}

We calculated four types of reproducibility: technical reproducibility, lab reproducibility, aligner reproducibility, and caller reproducibility.

A reproducibility value was calculated between two sets a ( $N^{a}$ variants) and b ( $N^{b}$ variants) using eval command of RTG Tools (v3.9). First, we indexed the reference genome to sdf format with RTG's format command. Then we took set a as querying and set $\mathrm{b}$ as baseline for the calculation. Four sets of variants were output from the calculation: unique variants in a, variants of a found in $\mathrm{b}\left(n^{a}\right)$, unique variants in $\mathrm{b}$, and variants of $\mathrm{b}$ found in a $\left(n^{b}\right)$. Basic information such as variant type and number was counted by RTG's stat command. All numbers were extracted with a shell script written to extract the numbers and to calculate reproducibility $\mathrm{R}$ using equation (1).

$$
R=\frac{1}{2}\left(\frac{n^{a}}{N^{a}}+\frac{n^{b}}{N^{b}}\right)
$$

\section{Calculation of precision, recall, and F-score}

Precision, recall, and F-score were calculated by comparing a set of variants with its corresponding HRVs using equations (2-4). The comparison was done using RTG's eval command. 
Precision $=\frac{Q^{c}}{Q^{c}+Q^{u}}$

Recall $=\frac{Q^{c}}{Q^{c}+H^{u}}$

$$
\mathrm{F}_{\text {score }}=2 * \text { Precsion } * \frac{\text { Recall }}{\text { Precision }+ \text { Recall }}
$$

where $Q^{c}$ is number of common variants; $Q^{u}$ is number of variants in the comparing set but not in the HRVs; and $H^{u}$ is number of variants in the HRVs but not in the comparing set.

\section{Supplementary Information}

The online version contains supplementary material available at https://doi.org/10.1186/s13059-021-02569-8.

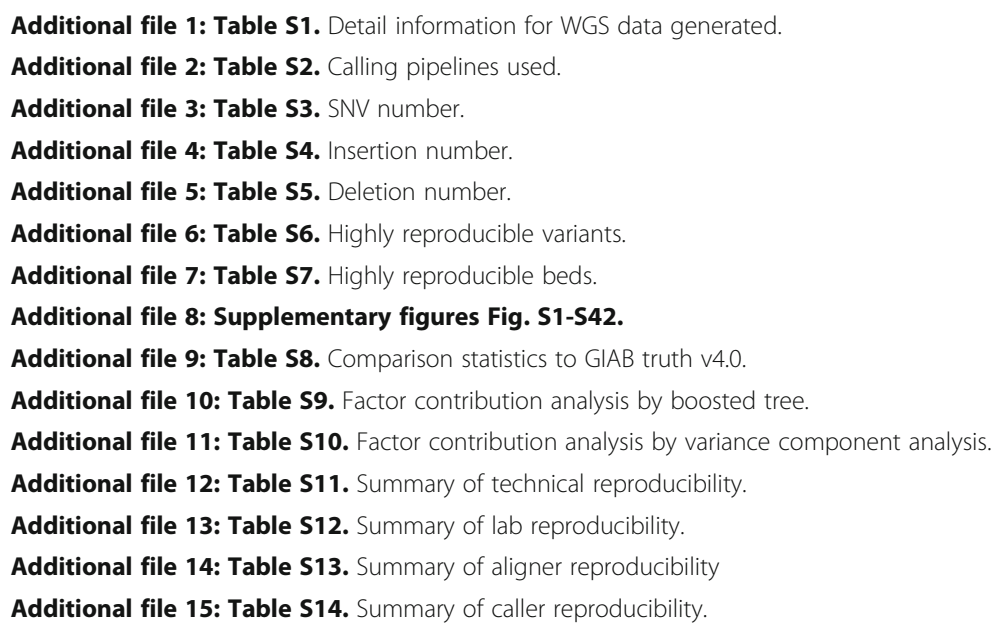

This manuscript was previously reviewed at another journal; no review history is available.

\section{Peer review information}

Barbara Cheifet was the primary editor of this article and managed its editorial process and peer review in collaboration with the rest of the editorial team.

\section{Disclaimer}

The content is solely the responsibility of the authors and does not necessarily represent the official views of U.S. Food and Drug Administration, National Institute of Standards and Technology, National Institutes of Health, and U.S. Army Corps of Engineers. Certain commercial equipment, instruments, or materials are identified to specify adequately experimental conditions or reported results. Such identification does not imply recommendation or endorsement by the National Institute of Standards and Technology, nor does it imply that the equipment, instruments, or materials identified are necessarily the best available for the purpose.

\section{Authors' contributions}

B.P., L.R., V.O., M.G., S.B., L.T., H.C., T.H., T.C., B.N., W.B., W.X., X.F., Y.G., and H.H. performed the experiments. B.P., L.R., V.O., M.G., S.B., L.T., H.C., T.H., X.C., G.Y., J.S., K.M., Y.Z., and H.H. analyzed the data. J.M., J.W., J.Y., W.G., W.H., T.S., T.H., Y.Y., W.Z., W.G., Y.Z., and L.S. provided materials. C.Z., C.G.K., E.D., A.S., J.M.Z., G.S., H.G., H.M., W.X., W.T., Z.L., Z.S., L.D., M.M., M.P., P.G., R.G., R.W., S.L., S.M.E.S., S.S., and L.S. conceived and oversaw the study. B.P., R.K., L.T., Y.Z., J.F., S.B., F.J.S., J.M.Z., and H.H. wrote the draft and all authors revised the manuscript. All author(s) read and approved the final manuscript.

\section{Funding}

Yuanting Zheng and Leming Shi were supported in part by the National Key R\&D Project of China (2018YFE0201603, 2018YFE0201600, and 2017YFF0204600), the National Natural Science Foundation of China (31720103909 and 32170657), and Shanghai Municipal Science and Technology Major Project (2017SHZDZX01), State Key Laboratory of Genetic Engineering (SKLGE-2117), and the 111 Project (B13016). Chunlin Xiao and Steve Sherry were supported by the Intramural Research Program of the National Library of Medicine, National Institutes of Health. Yoichi Gondo was partly supported by JSPS KAKENHI no. 17H00789. Tieliu Shi was supported by the Open Research Fund of Key 
Laboratory of Advanced Theory and Application in Statistics and Data Science -MOE, ECNU and Shanghai Municipal Science and Technology Major Project (2017SHZDZX01). Fritz J. Sedlazeck was supported by NIH (UM1 HG008898).

\section{Availability of data and materials}

All raw read data (FASTQ files) are available in the National Omics Data Encyclopedia (NODE) database (https://www. biosino.org/node) and the SRA database (https://www.ncbi.nlm.nih.gov/sra). The Chinese Quartet data are available in NODE with the accession number OEP001896 [64]. The HapMap Trio data are available in SRA with the accession number PRJNA723125 [65]. High reproducible sets are available at Zenodo (https://zenodo.org/record/5275189\#. YaaYn9DMJPZ) [66]. All codes used in processing the WGS data and reproducibility calculation are available at Github (https://github.com/justwalking2017/SEQC_WG3_Script) [67].

\section{Declarations}

\section{Ethics approval and consent to participate}

This Quartet project was approved by the Institutional Review Board (IRB) of School of Life Sciences, Fudan University (BE2049). All the four donors have signed informed consents. The HapMap trio and NA12878 sample come from purchasing of commercial cell line that do not require an approval or consent to participate from human donors. All experimental methods comply with the Helsinki Declaration.

\section{Competing interests}

The authors declare that they have no competing interests.

\section{Author details}

'Division of Bioinformatics and Biostatistics, National Center for Toxicological Research, US Food and Drug Administration, Jefferson, AR 72079, USA. ${ }^{2}$ State Key Laboratory of Genetic Engineering, School of Life Sciences and Shanghai Cancer Center, Fudan University, Shanghai 200438, China. ${ }^{3}$ Human Phenome Institute, Fudan University, Shanghai 200438, China. ${ }^{4}$ Illumina Inc., San Diego, CA 92122, USA. ${ }^{5}$ SAS Institute Inc., Cary, NC 27513, USA. ${ }^{6}$ Immuneering Corporation, Cambridge, MA 02142, USA. ${ }^{7}$ Real Time Genomics, Hamilton, New Zealand. ${ }^{8}$ Institute for Molecular Medicine Finland (FIMM), University of Helsinki, Helsinki, Finland. ${ }^{9}$ EATRIS ERIC- European Infrastructure for Translational Medicine, Amsterdam, the Netherlands. ${ }^{10}$ School of Computing Sciences and Computer Engineering, University of Southern Mississippi, Hattiesburg, MS 39406, USA. ${ }^{11}$ National Center for Biotechnology Information, National Library of Medicine, National Institutes of Health, Bethesda, MD 20894, USA. ${ }^{12}$ Center for Drug Evaluation and Research, Food and Drug Administration, Silver Spring, MD 20993, USA. ${ }^{13}$ Human Genome Sequencing Center, Baylor College of Medicine, Houston, TX 77030, USA. ${ }^{14}$ Sentieon Inc., San Jose, CA 95134, USA. ${ }^{15}$ Center for Advanced Measurement Science, National Institute of Metrology, Beijing 100013, China. ${ }^{16}$ Department of Physiology and Biophysics, Weill Cornell Medicine, New York, NY 10021, USA. ${ }^{17}$ Roche Sequencing Solutions, Santa Clara, CA 95050, USA. ${ }^{18}$ Bioinformatics and Computational Biology Laboratory, National Heart Lung and Blood Institute, National Institutes of Health, Bethesda, MD 20892, USA. ${ }^{19}$ Environmental Laboratory, U.S. Army Engineer Research and Development Center, Vicksburg, MS 39180, USA. ${ }^{20}$ Office of Health Informatics, Office of the Commissioner, US Food and Drug Administration, Silver Spring, MD 20993, USA. ${ }^{21}$ The Center for Bioinformatics and Computational Biology, Shanghai Key Laboratory of Regulatory Biology, Institute of Biomedical Sciences and School of Life Sciences, East China Normal University, Shanghai 200241, China. ${ }^{22}$ Stanford Genome Technology Center, Stanford University School of Medicine, Palo Alto, CA 94305, USA. ${ }^{23}$ Pharmaceutical Informatics Institute, College of Pharmaceutical Sciences, Zhejiang University, Hangzhou 310058, China. ${ }^{24}$ Department of Molecular Life Sciences, Tokai University School of Medicine, 143 Shimokasuya, Isehara 259-1193, Japan. ${ }^{25}$ CCR-SF Bioinformatics Group, Advanced Biomedical and Computational Sciences, Biomedical Informatics and Data Science, Frederick National Laboratory for Cancer Research, Frederick, MD 21701, USA. ${ }^{26}$ Takeda Pharmaceuticals, Cambridge, MA 02139, USA. ${ }^{27}$ Division of Molecular Genetics and Pathology, Center for Device and Radiological Health, US Food and Drug Administration, Silver Spring, MD 20993, USA.

${ }^{28}$ Material Measurement Laboratory, National Institute of Standards and Technology, Gaithersburg, MD 20899, USA.

Received: 22 November 2021 Accepted: 6 December 2021

Published online: 03 January 2022

\section{References}

1. Cheng DT, Prasad M, Chekaluk Y, Benayed R, Sadowska J, Zehir A, et al. Comprehensive detection of germline variants by MSK-IMPACT, a clinical diagnostic platform for solid tumor molecular oncology and concurrent cancer predisposition testing. BMC Med Genomics. 2017:10(1):33. https://doi.org/10.1186/s12920-017-0271-4.

2. Smith IN, Thacker S, Seyfi M, Cheng F, Eng C. Conformational dynamics and allosteric regulation landscapes of germline PTEN mutations associated with autism compared to those associated with cancer. Am J Hum Genet. 2019;104(5):86178. https://doi.org/10.1016/j.jhg.2019.03.009.

3. Din S, Wong K, Mueller MF, Oniscu A, Hewinson J, Black CJ, et al. Mutational analysis identifies therapeutic biomarkers in inflammatory bowel disease-associated colorectal cancers. Clin Cancer Res. 2018;24(20):5133-42. https://doi.org/10.11 58/1078-0432.CCR-17-3713.

4. Haapaniemi EM, Kaustio M, Rajala HL, van Adrichem AJ, Kainulainen L, Glumoff V, et al. Autoimmunity, hypogammaglobulinemia, lymphoproliferation, and mycobacterial disease in patients with activating mutations in STAT3. Blood. 2015;125(4):639-48. https://doi.org/10.1182/blood-2014-04-570101.

5. Wright GEB, Collins JA, Kay C, McDonald C, Dolzhenko E, Xia Q, et al. Length of uninterrupted CAG, independent of polyglutamine size, results in increased somatic instability, hastening onset of Huntington disease. Am J Hum Genet. 2019;104(6):1116-26. https://doi.org/10.1016/j.ajhg.2019.04.007. 
6. Andrews KA, Ascher DB, Pires DEV, Barnes DR, Vialard L, Casey RT, et al. Tumour risks and genotype-phenotype correlations associated with germline variants in succinate dehydrogenase subunit genes SDHB, SDHC and SDHD. J Med Genet. 2018;55(6):384-94. https://doi.org/10.1136/jmedgenet-2017-105127.

7. Davies JC, Wainwright CE, Canny GJ, Chilvers MA, Howenstine MS, Munck A, et al. Efficacy and safety of ivacaftor in patients aged 6 to 11 years with cystic fibrosis with a G551D mutation. Am J Respir Crit Care Med. 2013;187(11):121925. https://doi.org/10.1164/rccm.201301-01530C.

8. Robasky K, Lewis NE, Church GM. The role of replicates for error mitigation in next-generation sequencing. Nat Rev Genet. 2014;15(1):56-62. https://doi.org/10.1038/nrg3655.

9. Li H. Toward better understanding of artifacts in variant calling from high-coverage samples. Bioinformatics. 2014;30(20): 2843-51. https://doi.org/10.1093/bioinformatics/btu356.

10. Sims D, Sudbery I, llott NE, Heger A, Ponting CP. Sequencing depth and coverage: key considerations in genomic analyses. Nat Rev Genet. 2014;15(2):121-32. https://doi.org/10.1038/nrg3642.

11. Heinrich V, Stange J, Dickhaus T, Imkeller P, Kruger U, Bauer S, et al. The allele distribution in next-generation sequencing data sets is accurately described as the result of a stochastic branching process. Nucleic Acids Res. 2012; 40(6):2426-31. https://doi.org/10.1093/nar/gkr1073.

12. Kishikawa T, Momozawa Y, Ozeki T, Mushiroda T, Inohara H, Kamatani Y, et al. Empirical evaluation of variant calling accuracy using ultra-deep whole-genome sequencing data. Sci Rep. 2019;9(1):1784. https://doi.org/10.1038/s41598-018-38346-0.

13. Fox EJ, Reid-Bayliss KS, Emond MJ, Loeb LA. Accuracy of next generation sequencing platforms. Next Gener Seq Appl. 2014;1(01). https://doi.org/10.4172/2469-9853.1000106.

14. Zook JM, Chapman B, Wang J, Mittelman D, Hofmann O, Hide W, et al. Integrating human sequence data sets provides a resource of benchmark SNP and indel genotype calls. Nat Biotechnol. 2014;32(3):246-51. https://doi.org/10.1038/nbt.2835.

15. Eberle MA, Fritzilas E, Krusche P, Kallberg M, Moore BL, Bekritsky MA, et al. A reference data set of 5.4 million phased human variants validated by genetic inheritance from sequencing a three-generation 17-member pedigree. Genome Res. 2017;27(1):157-64. https://doi.org/10.1101/gr.210500.116.

16. Li H, Bloom JM, Farjoun Y, Fleharty M, Gauthier L, Neale B, et al. A synthetic-diploid benchmark for accurate variantcalling evaluation. Nat Methods. 2018;15(8):595-7. https://doi.org/10.1038/s41592-018-0054-7.

17. Krusche P, Trigg L, Boutros PC, Mason CE, De La Vega FM, Moore BL, et al. Best practices for benchmarking germline small-variant calls in human genomes. Nat Biotechnol. 2019;37(5):555-60. https://doi.org/10.1038/s41587-019-0054-X.

18. Chen J, Li X, Zhong H, Meng Y, Du H. Systematic comparison of germline variant calling pipelines cross multiple nextgeneration sequencers. Sci Rep. 2019;9(1):9345. https://doi.org/10.1038/s41598-019-45835-3.

19. Patch AM, Nones K, Kazakoff SH, Newell F, Wood S, Leonard C, et al. Germline and somatic variant identification using BGISEQ-500 and HiSeq X Ten whole genome sequencing. PLoS One. 2018;13(1):e0190264. https://doi.org/10.1371/journa I.pone.0190264.

20. Lam HY, Clark MJ, Chen R, Chen R, Natsoulis G, O'Huallachain M, et al. Performance comparison of whole-genome sequencing platforms. Nat Biotechnol. 2011;30(1):78-82. https://doi.org/10.1038/nbt.2065.

21. O'Rawe J, Jiang T, Sun G, Wu Y, Wang W, Hu J, et al. Low concordance of multiple variant-calling pipelines: practical implications for exome and genome sequencing. Genome Med. 2013;5(3):28. https://doi.org/10.1186/gm432.

22. Hwang KB, Lee IH, Li H, Won DG, Hernandez-Ferrer C, Negron JA, et al. Comparative analysis of whole-genome sequencing pipelines to minimize false negative findings. Sci Rep. 2019;9(1):3219. https://doi.org/10.1038/s41598-019-39108-2.

23. International HapMap C. The International HapMap Project. Nature. 2003;426(6968):789-96. https://doi.org/10.1038/nature02168.

24. Zook JM, Catoe D, McDaniel J, Vang L, Spies N, Sidow A, et al. Extensive sequencing of seven human genomes to characterize benchmark reference materials. Sci Data. 2016;3(1):160025. https://doi.org/10.1038/sdata.2016.25.

25. Khayat M, Sahraeian SME, Zarate S, Carroll A, Hong H, Pan B, et al. Genome Biol. 2021.

26. Lander ES, Linton LM, Birren B, Nusbaum C, Zody MC, Baldwin J, et al. Initial sequencing and analysis of the human genome. Nature. 2001;409(6822):860-921.

27. Zhang W, Ng HW, Shu M, Luo H, Su Z, Ge W, et al. Comparing genetic variants detected in the 1000 genomes project with SNPs determined by the International HapMap Consortium. J Genet. 2015;94(4):731-40. https://doi.org/10.1007/s12 041-015-0588-8.

28. Lercher MJ, Urrutia AO, Pavlicek A, Hurst LD. A unification of mosaic structures in the human genome. Hum Mol Genet. 2003;12(19):2411-5. https://doi.org/10.1093/hmg/ddg251.

29. Ludwig MZ. Functional evolution of noncoding DNA. Curr Opin Genet Dev. 2002;12(6):634-9. https://doi.org/10.1016/ S0959-437X(02)00355-6.

30. Zook JM, McDaniel J, Olson ND, Wagner J, Parikh $\mathrm{H}$, Heaton $\mathrm{H}$, et al. An open resource for accurately benchmarking small variant and reference calls. Nat Biotechnol. 2019;37(5):561-6. https://doi.org/10.1038/s41587-019-0074-6.

31. Olson ND, Wagner J, McDaniel J, Stephens SH, Westreich ST, Prasanna AG, et al. precisionFDA Truth Challenge V2: calling variants from short- and long-reads in difficult-to-map regions. bioRxiv. 2021; 2020.2011.2013.380741.

32. Hatem A, Bozdag D, Toland AE, Catalyurek UV. Benchmarking short sequence mapping tools. BMC Bioinformatics. 2013; 14(1):184. https://doi.org/10.1186/1471-2105-14-184.

33. Fonseca NA, Rung J, Brazma A, Marioni JC. Tools for mapping high-throughput sequencing data. Bioinformatics. 2012; 28(24):3169-77. https://doi.org/10.1093/bioinformatics/bts605.

34. Linderman MD, Brandt T, Edelmann L, Jabado O, Kasai Y, Kornreich R, et al. Analytical validation of whole exome and whole genome sequencing for clinical applications. BMC Med Genomics. 2014;7(1):20. https://doi.org/10.1186/1755-8794-7-20.

35. Van der Auwera GA, Carneiro MO, Hartl C, Poplin R, Del Angel G, Levy-Moonshine A, et al. From FastQ data to high confidence variant calls: the Genome Analysis Toolkit best practices pipeline. Curr Protoc Bioinformatics. 2013:43:11 10 11-11 1033.

36. Povysil G, Petrovski S, Hostyk J, Aggarwal V, Allen AS, Goldstein DB. Rare-variant collapsing analyses for complex traits: guidelines and applications. Nat Rev Genet. 2019;20(12):747-59. https://doi.org/10.1038/s41576-019-0177-4.

37. Gutierrez-Arcelus M, Rich SS, Raychaudhuri S. Autoimmune diseases - connecting risk alleles with molecular traits of the immune system. Nat Rev Genet. 2016;17(3):160-74. https://doi.org/10.1038/nrg.2015.33.

38. Chat V, Ferguson R, Simpson D, Kazlow E, Lax R, Moran U, et al. Autoimmune genetic risk variants as germline biomarkers of response to melanoma immune-checkpoint inhibition. Cancer Immunol Immunother. 2019;68(6):897-905 https://doi.org/10.1007/s00262-019-02318-8. 
39. Rana HQ, Sacca R, Drogan C, Gutierrez S, Schlosnagle E, Regan MM, et al. Prevalence of germline variants in inflammatory breast cancer. Cancer. 2019;125(13):2194-202. https://doi.org/10.1002/cncr.32062.

40. Altmüller F, Lissewski C, Bertola D, Flex E, Stark Z, Spranger S, et al. Genotype and phenotype spectrum of NRAS germline variants. Eur J Hum Genet. 2017;25(7):823-31. https://doi.org/10.1038/ejhg.2017.65.

41. Pagnamenta AT, Murakami Y, Taylor JM, Anzilotti C, Howard MF, Miller V, et al. Analysis of exome data for 4293 trios suggests GPI-anchor biogenesis defects are a rare cause of developmental disorders. Eur J Hum Genet. 2017;25(6):66979. https://doi.org/10.1038/ejhg.2017.32.

42. Earl J, Galindo-Pumariño C, Encinas J, Barreto E, Castillo ME, Pachón V, et al. Ramon y Cajal T, et al: A comprehensive analysis of candidate genes in familial pancreatic cancer families reveals a high frequency of potentially pathogenic germline variants. EBioMedicine. 2020;53:102675. https://doi.org/10.1016/j.ebiom.2020.102675.

43. Pan B, Kusko R, Xiao W, Zheng Y, Liu Z, Xiao C, et al. Similarities and differences between variants called with human reference genome HG19 or HG38. BMC Bioinformatics. 2019;20(S2):101. https://doi.org/10.1186/s12859-019-2620-0.

44. Treangen TJ, Salzberg SL. Repetitive DNA and next-generation sequencing: computational challenges and solutions. Nat Rev Genet. 2012;13(1):36-46. https://doi.org/10.1038/nrg3117.

45. Ebbert MTW, Jensen TD, Jansen-West K, Sens JP, Reddy JS, Ridge PG, et al. Systematic analysis of dark and camouflaged genes reveals disease-relevant genes hiding in plain sight. Genome Biol. 2019;20(1):97. https://doi.org/10.1186/s13059-019-1707-2.

46. Marx V. Bench pressing with genomics benchmarkers. Nat Methods. 2020;17(3):255-8. https://doi.org/10.1038/s41592-020-0768-1.

47. Wenger AM, Peluso P, Rowell WJ, Chang P-C, Hall RJ, Concepcion GT, et al. Accurate circular consensus long-read sequencing improves variant detection and assembly of a human genome. Nat Biotechnol. 2019;37(10):1155-62. https://doi.org/10.1038/s41587-019-0217-9.

48. Sedlazeck FJ, Rescheneder P, Smolka M, Fang H, Nattestad M, von Haeseler A, et al. Accurate detection of complex structural variations using single-molecule sequencing. Nat Methods. 2018;15(6):461-8. https://doi.org/10.1038/s41592018-0001-7.

49. Xiao W, Ren L, Chen Z, Fang LT, Zhao Y, Lack J, Guan M, Zhu B, Jaeger E, Kerrigan L, Blomquist TM, Hung T, Sultan M, Idler K, Lu C, Scherer A, Kusko R, Moos M, Xiao C, Sherry ST, Abaan OD, Chen W, Chen X, Nordlund J, Liljedahl U, Maestro R, Polano M, Drabek J, Vojta P, Köks S, Reimann E, Madala BS, Mercer T, Miller C, Jacob H, Truong T, Moshrefi A, Natarajan A, Granat A, Schroth GP, Kalamegham R, Peters E, Petitjean V, Walton A, Shen TW, Talsania K, Vera CJ, Langenbach K, de Mars M, Hipp JA, Willey JC, Wang J, Shetty J, Kriga Y, Raziuddin A, Tran B, Zheng Y, Yu Y, Cam M, Jailwala P, Nguyen C, Meerzaman D, Chen Q, Yan C, Ernest B, Mehra U, Jensen RV, Jones W, Li JL, Papas BN, Pirooznia M, Chen YC, Seifuddin F, Li Z, Liu X, Resch W, Wang J, Wu L, Yavas G, Miles C, Ning B, Tong W, Mason CE, Donaldson E, Lababidi S, Staudt LM, Tezak Z, Hong H, Wang C, Shi L. Toward best practice in cancer mutation detection with wholegenome and whole-exome sequencing. Nat Biotechnol. 2021:39(9):1141-50. https://doi.org/10.1038/s41587-021-00994-5.

50. Amarasinghe SL, Su S, Dong X, Zappia L, Ritchie ME, Gouil Q. Opportunities and challenges in long-read sequencing data analysis. Genome Biol. 2020;21(1):30. https://doi.org/10.1186/s13059-020-1935-5.

51. Andrews S: FastQC: a quality control tool for high throughput sequence data. Available online at: http://www. bioinformaticsbabrahamacuk/projects/fastqc/ 2010.

52. Langmead B, Salzberg SL. Fast gapped-read alignment with Bowtie 2. Nat Methods. 2012;9(4):357-9. https://doi.org/10.1 038/nmeth.1923.

53. Li H. Aligning sequence reads, clone sequences and assembly contigs with BWA-MEM; 2013.

54. Raczy C, Petrovski R, Saunders CT, Chorny I, Kruglyak S, Margulies EH, et al. Isaac: ultra-fast whole-genome secondary analysis on Illumina sequencing platforms. Bioinformatics. 2013;29(16):2041-3. https:/doi.org/10.1093/bioinformatics/btt314.

55. Lunter G, Goodson M. Stampy: a statistical algorithm for sensitive and fast mapping of Illumina sequence reads. Genome Res. 2011;21(6):936-9. https://doi.org/10.1101/gr.111120.110.

56. Tamminga CA. The human genome sequence: the human genome I: chromosomes and protein coding. Am J Psychiatry. 2001;158(3):370. https://doi.org/10.1176/appi.ajp.158.3.370.

57. Garrison EM. G: Haplotype-based variant detection from short-read sequencing; 2012.

58. Li H, Handsaker B, Wysoker A, Fennell T, Ruan J, Homer N, et al. The Sequence Alignment/Map format and SAMtools. Bioinformatics. 2009;25(16):2078-9. https://doi.org/10.1093/bioinformatics/btp352.

59. Wei Z, Wang W, Hu P, Lyon GJ, Hakonarson H. SNVer: a statistical tool for variant calling in analysis of pooled or individual next-generation sequencing data. Nucleic Acids Res. 2011;39(19):e132. https://doi.org/10.1093/nar/gkr599.

60. Koboldt DC, Zhang Q, Larson DE, Shen D, McLellan MD, Lin L, et al. VarScan 2: somatic mutation and copy number alteration discovery in cancer by exome sequencing. Genome Res. 2012;22(3):568-76. https://doi.org/10.1101/gr.129684.111.

61. Freed D, Aldana R, Weber JA, Edwards JS. The Sentieon Genomics Tools - a fast and accurate solution to variant calling from next-generation sequence data. bioRxiv. 1157172017.

62. Cleary JG, Braithwaite R, Gaastra K, Hilbush BS, Inglis S, Invine SA, Jackson A, Littin R, Rathod M, Ware D, Zook JM, Trigg L, De La Vega FM. Haplotype-based variant detection from short-read sequencing. BioRxiv. 2015. https://doi.org/10.1101/023754.

63. Cobb GW. Introduction to design and analysis of experiments. Hoboken, New Jersey: Wiley; 2008.

64. Pan, B, Ren L, Onuchic V, Guan M, Kusko R, Hong H, et al. Assessing reproducibility of inherited variants detected with short-read whole genome sequencing. NODE. biosino.org/node/project/detail/OEP001896. Accessed 1 Dec 2021.

65. Pan, B, Ren L, Onuchic V, Guan M, Kusko R, Hong H, et al. Assessing reproducibility of inherited variants detected with shortread whole genome sequencing. SRA. https://www.ncbinlm.nih.gov/bioproject/PRJNA723125. Accessed 1 Dec 2021.

66. Pan B, Ren L, Onuchic V, Guan M, Kusko R, Hong H, et al. Assessing reproducibility of inherited variants detected with short-read whole genome sequencing. Zenodo. 2021. https://doi.org/10.5281/zenodo.5275189.

67. Pan B, Ren L, Onuchic V, Guan M, Kusko R, Hong H, et al. Assessing reproducibility of inherited variants detected with shortread whole genome sequencing. Github. https://github.com/justwalking2017/SEQC_WG3_Script. Accessed 1 Dec 2021.

\section{Publisher's Note}

Springer Nature remains neutral with regard to jurisdictional claims in published maps and institutional affiliations. 